\title{
An Efferent Component in the Visual Perception of Direction and Extent
}

\author{
Stanley Coren \\ University of British Columbia, Vancouver, British Columbia, Canada
}

\begin{abstract}
After outlining the history of motor theories of visual perception, a new theory linking information extraction patterns, specifically adapted for the guidance of eye movements, to the visual perception of direction and extent is presented. Following a brief discussion of comparative and physiological considerations, a research strategy to test for efferent involvement in visual perception in humans is presented. In seven demonstration experiments, predictions from efferent considerations are used to create a new set of illusions of direction and extent and to demonstrate new predictable variations in the magnitude of some classical illusion figures. Another demonstration illustrates that systematic changes in visual perception occur as a function of changes in motoric demands, even in the absence of any configurational changes in the stimulus. A final section shows the relationship between attention and efferent readiness and their interaction in the formation of the conscious visual percept.
\end{abstract}

From a historical perspective, most contemporary theories of visual perception are quite conservative. This conservatism springs from an apparent acceptance of the premise that any proper analysis of visual experience must avoid reference to nonvisual mechanisms, except for labeling and semantic aspects of the perceptual process. It follows that most visual theorists tend to derive virtually every aspect of the conscious percept solely from either the physical characteristics of the visual stimulus array or the operation of readily definable neurological units in the visual system.

Characteristic of the former viewpoint is Gibson's (1979) theory of ecological optics, which maintains that virtually all aspects of the final percept are predictable from invariants in the stimulus array. Current attempts to derive the conscious percept from a hypothesized Fourier analysis occurring within the visual system are similar in approach, merely relying on higher level processing of the physical stimulus (e.g., Weisstein \& Harris, 1980). Using the current literature as an index, the only seemingly acceptable alternative to derivation of the final percept directly from physical stimulus properties is to adopt a neuroreductionist approach. Here the investigator is expected to isolate specific neural units or channels that are then held to account for each aspect of the the final subjective percept (e.g., Graham, Robson, \& Nachmias, 1978; Hubel, 1978). Although the above-mentioned approaches have a certain allure, providing concrete mechanisms and clearly calculable stimulus parameters on which to rest conclusions, there are some alternative theoretical treatments of visual perception, which do allow

This research was supported in part by grants from the National Science and Engineering Research Council of Canada.

Too many people have helped shape the ideas contained in this article to be acknowledged separately. However, two who deserve special thanks are Clare Porac and Joan S. Girgus.

Correspondence concerning this article should be addressed to Stanley Coren, Department of Psychology, University of British Columbia, Vancouver, British Columbia V6T 1W5, Canada. contribution from nonvisual sources, that seem to have slipped from contemporary collective consciousness. At least one of these alternatives, based on motoric or efferent contributions to the final percept, may deserve a second look.

\section{Early Efferent Theories of Perception}

In contrast to our modern approach to visual perception, the earliest treatments of sensory experience did not make a distinction between the visual and the nonvisual components involved in the process of seeing. Thus, well before 1855 , when Alexander Bain published the first English textbook on the psychology of perception, theorists had been toying with the notion that sensory and motor functions might be part of a unified system working in conjunction to form the conscious percept. The nature of this sensory-motor interaction has always been somewhat obscure. Be that as it may, because eye movements are clearly made in response to perceptually relevant inputs, they were naturally singled out as the motoric responses most likely to interact in the formation of the visual percept.

Bain (1855) suggested that "by a horizontal sweep, we take in a horizontal line; by a circular sweep, we derive the muscular impression of a circle" (p. 236). He goes on to state that "muscular consciousness" is an indispensable component in the formation of the percept. Thus, "a circle is a series of ocular movements" (p. 373) and "naked outlines, as the diagrams of Euclid and the alphabetical characters, are to say the least of it, three parts muscular and one part optical." In so doing, Bain proposes a motor theory that reduces all conscious visual perception to a set of eye movements.

Wundt's psychological research program, conducted during this same era, was influenced by this ambient theoretical zeitgeist. He was aware of Oppel's (1854-1855) analysis of several visual-geometric illusions, specifically the Oppel-Kundt illusion (see Figure 1A), where the upper divided extent looks longer than the lower undivided extent, and the horizontal-vertical illusion (see Figure 1B), where the vertical line appears 


\section{$|I| I|I| \mid$}

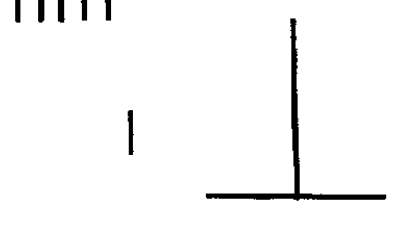

A

Figure 1. A: Oppel-Kundt illusion. B: Horizontal-vertical illusion. C and D: Mueller-Lyer illusion.

longer than the horizontal. Wundt incorporated an efferent component in his 1897 analysis of the source of these illusory distortions:

The phenomena of vision teach that the idea of the relative distance of two points from each other is dependent on the motor energy of the eye employed in passing through this distance. . . . The motor energy becomes a component of the idea through its connection with a sensation which can be perceived." (p. 133; emphasis in the original)

Wundt's argument is based on the trivial observation that it takes a longer eye movement to traverse a long line in the visual field than to traverse a shorter line. Because the amount of effort expended in making eye movements is proportional to the length of the stimulus, Wundt suggested that some sort of feedback indicating the amount of muscular effort expended is incorporated into perceptual estimates of length. One should not underestimate the cleverness of this approach because it predicts that anything that alters the effort needed to make an eye movement over a stimulus should alter the estimate of the size of that stimulus. Wundt directly applied this principle to explain several illusions.

Introspection convinced Wundt that horizontal movements of the eye are apparently more freely and easily made (with less subjective feeling of effort) than are vertical eye movements, which must fight the force of gravity. If different amounts of motoric effort are needed for these two classes of movement, why vertical lines are seen as longer than horizontal lines of equal length, as in the horizontal-vertical illusion (depicted in Figure 1B), might be explained. The argument is that a vertical extent of the same physical length as a horizontal extent is perceived as being longer merely because it elicits eye movements requiring more effort. Wundt extended this line of reasoning to explain the Oppel-Kundt illusion (Figure 1A). He suggested that in the segment with divided space the eye has a tendency to stop occasionally on some of the interior elements. Such stopping and starting requires more effort than a single movement across the open extent; thus, a simple application of the principle that the amount of effort expended interacts with the visual perception of extent allows the prediction that the divided space should be seen as longer, even though the two physical extents are equal.

\section{Proprioceptive Feedback Theories}

A number of Wundt's contemporaries altered the basic concept of motoric involvement so that it could operate via the more objective concept of proprioceptive feedback from emitted movements rather than on Wundt's more subjective "feeling of effort." Visual-geometric illusions were held to be the prototypical demonstration of the validity of this idea. Here the notion was that particular stimulus arrays induce some form of erroneous eye movement on the part of the observer. The proprioception from these erroneous eye movements in turn enters into and distorts the conscious perception of the stimulus pattern. Thus Binet (1895) and van Bierveliet (1896) in France, Lipps (1897) in Germany, and Judd (1905) in the United States each independently proposed that the Mueller-Lyer illusion (see Figures IC and 1D) could be explained by this process. The general argument suggests that the eyes tend to be drawn beyond the vertex into the outwardly turned wings (Figure $1 \mathrm{C}$ ) or, conversely, arrested by the inwardly turned wings before they reach the end of the line (Figure 1D). Incorporation of the proprioceptive feedback from these erroneously long and short eye movements then easily constitutes an explanation for the obtained over- and underestimations of the linear extents.

These theoretical speculations stimulated a desire to actually measure eye movement patterns over various illusion configurations because they provide conditions where the observer's conscious percept differs from what might be expected on the basis of the actual physical measurements. If motoric factors are involved in the genesis of these distortions, then systematic eye movement errors should accompany the viewing of illusion configurations. The first empirical measurements of this type resulted from a heroic study by Delabarre (1897). His method of eye movement measurement involved attachment of a plaster cap to his eye and thence to a set of levers that mechanically recorded his eye movements while he viewed the Mueller-Lyer illusion. His results anticipated those of later researchers who had the advantage of photographic or bioelectric methods of recording. In general he found that the eye movements emitted while scanning the apparently longer segment were longer than those over the apparently shorter segment, as the theory predicts. This finding has been replicated many times (DeSisto \& Moses, 1968; Judd, 1905; Stratton, 1906; Yarbus, 1967). Perhaps the strongest confirmation comes from Festinger, White, and Allyn (1968), who observed that the size of the eye movement errors actually predicted the individual differences in the magnitude of the perceptual illusion.

Although the general pattern of data seems consistent with the original hypothesis of a motoric component in the perception of visual extent, at least for illusory stimuli, there are some disturbing points that are hard to explain. Consider the data from studies on the Mueller-Lyer figure as an example. Whereas the original saccadic eye movement is erroneously long or short, as predicted, most studies find that the eye shows a tendency to immediately rectify its error with a corrective flick in the appropriate direction. This correction results in a precise centering of the fovea on the intended vertex. Certainly if the percept is based on feedback from the actual eye movements emitted, these corrective flicks ought to be added into (or subtracted from) the estimate of length. Because the fovea eventually does rest accurately on the vertex, even though it has taken two eye movements to get there, the proprioceptive feedback should actually provide a fairly accurate estimate of the length of the line rather than any illusory effect. 
Further problems with the attempt to link proprioception from eye movements to the perception of extent or direction soon emerged. For instance, again using visual illusions as the model, one series of investigations attempted to eliminate the effects of eye movements by flashing stimuli too briefly for such movements to be made over the pattern. Despite the fact that the presentations were too brief to allow effective saccades to occur, the expected illusory distortions still occurred (Cooper \& Weintraub, 1970; Hicks \& Rivers, 1908; Lewis, 1909; Piaget \& Bang, 1961; Pollack, 1970). A similar rationale lay behind the use of the stabilized image technique, where optical systems or afterimage techniques are used to immobilize an image on the retina, rendering it stationary regardless of any eye movements. Despite the decorrelation between eye movements and image changes, most of the classical illusory effects are still observed (Ditchburn \& Ginsborg, 1952; Evans \& Marsden, 1966; Pritchard, 1958; Yarbus, 1967).

\section{Implicit Movement Theories}

At first glance the above data seem to negate any theory based on the supposition that there is a motoric contribution, at least in the form of eye movements, involved in the formation of visual illusions. However, it should be noted that, although these procedures do disrupt the normal relationship between eye movements and subsequent retinal image changes, they do not physically stop the eye from moving. Eye movements may still occur in the same direction, and even to the same extent, that they would occur under normal scanning conditions. In fact, there is evidence that even after a briefly presented image has disappeared, the saccadic eye movements are emitted on the basis of information extracted from the pattern during the brief period of time it was actually available for viewing (Crovitz \& Davies, 1962). A strict interpretation of the efferent contribution to the perception of extent would hold that as long as such eye movements occur, whether or not they are effective in actually scanning over the stimulus, the proprioceptive record of the extent of the emitted eye movement could be entered into the final computation of perceived extent. Furthermore, if the size of these ineffective eye movements is systematically biased, the movements could still result in biased proprioceptive feedback and, hence, the perception of an illusion.

This version of an efferent theory received further support form an unexpected source. The newly emergent behaviorist school of psychology evinced a marked fondness for motor theories of perception. These early behaviorists altered the theory to make it more consistent with the data (albeit less empirically accessible to direct testing) by doing away with the necessity for actual eye movements and gross proprioceptive feedback. Thus, Washburn (1916) maintained that although overt eye movements are certainly necessary for developing young organisms to originally establish spatial and directional percepts, at later stages of development these manifest movements are unnecessary. Instead the percept is supported by implicit, partial components of the movements. These implicit movements were held to form the basis of all conscious thought, as well as perception (Jacobson, 1938; Max, 1935; Skinner, 1974; Watson, 1930). In effect, these formulations reduce perception to feed- back from covert muscle twitches rather than overt or visible movements.

Implicit movement involvement in perception can be directly tested with the aid of neural muscular blocks, such as curare. These drugs make it possible to eliminate even these tiny muscular twitches, by blocking transmission across the neural muscular junction. Perhaps the most spectacular experiment along this line is that done by Smith, Brown, Toman, and Goodman (1947), who were concerned by reports that patients who had been administered curare as an anesthetic not only felt pain but also could report events and conversations that occurred during the operation. Smith, serving as the subject, was administered a dose of d-tubocurarine and reported that "at no time was there any evidence of lapses of consciousness or clouding of the sensorium" (p. 7). This statement was based on the fact that at intervals of a minute or less, during the period when communication with the subject was impossible owing to paralysis, stimuli were presented or objects placed in front of the line of gaze. The subject was requested to report these stimuli when speech returned. The investigators summarized the results by saying that "in each instance, the report was accurate in all details" (Smith et al., 1947, p. 7). Here we have perception of visual properties in the absence of feedback from any muscular response: overt, implicit, verbal, or otherwise.

\section{Efferent Readiness Theories}

There is an extremely subtle variant of a motor theory of perception that seems to circumvent some of the problems associated with both the overt and covert movement formulations. This conceptualization does not require any efference to be actually issued at all; rather it proposes that a set of eye movements are computed and held in readiness to be emitted across the visual array. The final conscious percept is synthesized from this set of efferent readinesses. A clear statement of this viewpoint is that of Muensterberg (1914), who contended that "we all perceive the world just as far as we are prepared to react to it. Our ability to respond is the true vehicle of our power to know" (p. 141). This alternate efferent readiness view of the relationship between the perception and the motoric system is historically of the same vintage as the overt response theory. There is a rather extensive discussion of it, as early as 1852 , in the work of Lotze. Hebb (1949) reformulated this position when he introduced the notion of the "phase sequence," which was a chain of central cortical events with motor links. He maintained that "although the motor activations may be subliminal and do not always produce overt response, their role is essential in any perception" (p. 35). The specific notion that the motoric response held in readiness might influence the final perception of extent or direction has sporadically reemerged in the literature in a number of different forms over the years (e.g., Festinger, Burnham, Ono, \& Bamber, 1967; Heymans, 1896; Sperry, 1952; Taylor, 1962). As is typical of most efferent theories of perception, one of its major uses has been as an explanatory device for a variety of visual illusions (e.g., Burnham, 1968; Coren, 1981; Coren \& Festinger, 1967; Coren \& Girgus, 1978a; Festinger, White, \& Allyn, 1968; Virsu, 1971).

Before the substantial methodological problem of how one begins to measure or manipulate a response held in readiness 
can be addressed, two philosophical issues must be resolved. First, if the organism already has enough information from which to compute the relevant efferent commands to overtly move the musculature, or even to hold these movements in readiness, why does it need to then refer back to such efferent components at all? Why does the system not simply use the sensory information that it has already obtained and processed (presumably in order to compute the efferent commands now held in readiness) as the basis for the conscious percept? Herein lies the major conceptual difficulty for any motor theory of perception. Second, even though it may be demonstrated that when an organism perceives a stimulus it also is ready to respond to it, there is still a question as to whether the resultant pattern of efference, either overt or held in readiness, is an integral part of the perceptual process or is merely a consequence of the perceptual process that has preceeded it. In terms of the illusion data, this asks simply whether the lines are seen as longer because the eye movement held in readiness is too long or the eye movement held in readiness is too long because the line already perceptually appears to be too long. To answer these questions the nature of the linkage between the perceptual and the motor processes must first be explored.

\section{Phylogenetic Arguments for a Motor Theory of Perception}

A basic postulate seems to lie at the heart of any motor theory of perception. This postulate is that the brain, viewed objectively, is primarily a mechanism for governing motor activity. Its raison d'être is the transformation of sensory patterns into patterns of motor coordination. This viewpoint is, of course, quite out of keeping with the generally accepted notion that the major functions of the brain are the manufacture of ideas, feelings, the storage of memory, and the interpretation of sensations into a conscious representation of the external environment. Such subjective phenomena, though salient to the behaving individual, may simply be epiphenomena-the byproduct of brain activity-rather than its targeted functional result. Few people would grant a fly, worm, or an octopus much in the way of feelings, ideas, or consciousness, yet each has something that may pass for a primitive brain and each responds adaptively with motoric activity to various sensory inputs. Even in higher organisms, except for the recordable electrical and chemical activities occurring within the cerebral vault, the entire activity of the brain, so far as has been yet determined, yields nothing but motor adjustment. When reduced to its essence, the fundamental interpretive task of the brain, whether direct or indirect, including the so-called "higher mental functions," is to transform the sensory inputs into motor programs that allow the organism to interact with the external environment. Despite the introspective salience of personal consciousness, the only means of expression or action available to the organism is through the efferent pathways and the motoric expression of behavior.

To psychologists, for whom the concept of consciousness still has an implicit (if now seldom mentioned) focal role in the understanding of higher behavioral functions, the interpretations of brain function given in the preceding paragraph probably seem to be extremely shortsighted, incomplete, and unsatisfactory. However, if a comparative or phylogenetic viewpoint is adopted, one will readily find data to support the contention that even in higher vertebrates the brain is designed primarily for the regulation of overt behavior rather than for some sort of mental performance. Thus, at the lower end of the phylogenetic scale, purely mental activity seems to be considerably less conspicuous in comparison with the amount of neural processing set aside for overt motoric response. In fact, to the extent that sensation or perception is manifest in many lower organisms, it appears to directly serve the function of guiding patterns of muscular contraction, or its equivalent. It is at this primitive level that the relationship between sensory and motor functions becomes most apparent.

The first evidence of any sensory ability in lower organisms appears to be in single-celled protozoans. The paramecium, for example, does not have any specialized reception apparatus; however, it shows a diffuse motoric reaction to light. In its presence, the paramecium increases its general activity level. It does not appear to swim purposefully in a given direction relative to the light stimuli, but it does swim at a faster pace. This results in a pattern of motor behavior that adaptively alters the relationship of the organism to the environment. For instance, suppose there is a pan of water with paramecium. A light is placed at one end of it, and the other end is shaded. After a while the animals will have crowded into the shaded end. This adaptive pattern of behavior is a consequence of a simple, automatic relationship between motoric output and incoming photic stimulation. Such direct changes in the activity level of an animal as a function of light input, as observed here, are called photokinesis. It not only provides evidence of an organism's sensitivity to light, but also provides an example of a situation where stimulation automatically results in activity changes. Thus the very first evidence of sentience to be found in any animal appears in the form of a rudimentary sensory-motor link.

In more phylogenetically advanced animals, specialized sensory cells begin crowding together to form receptors. At this level of development, a new sensory-motor response pattern begins to emerge. Now an animal will swim or orient itself, apparently purposefully, toward a light source, displaying what is commonly called phototaxis. Conversely, some animals may orient themselves away from the light, displaying a negative phototaxis. These responses are clearly apparent in flatworms, such as planaria, and in some animals as highly advanced as the annalids or segmented worms. These responses to stimuli are still quite automatic. The animal orients or moves reflexively according to the direction of the light source, and one may predict with almost perfect certainty the orientation of the organism on the basis of the knowledge of the location of the light source. The importance of such taxic behavior, however, is that it shows further evolution of the perceptual response, yet still demonstrates the direct driving of the motoric system by sensory inputs.

The lowly firefly provides the next stage of elaboration in the linkage between the motor system and perception. Suppose a female firefly detects the presence of a male. She raises her abdomen and emits a single burst of light. The male now turns as much as $180^{\circ}$ and flies to the place where the flash of light appeared. The essence of this act is that, although this response is just as automatic as the taxic responses observed above, this organism is flying toward a stimulus that is no longer physically 
present. This type of response may be called a nmenotaxis. It indicates that, although the sensory and motor systems are still inexorably bound in this animal, motoric responses to a trace or registration of the stimulus, rather than the stimulus itself, is the key factor. To be more accurate, the animal has extracted some information from the sensory array, and that extracted information is now driving its motor response pattern.

In vertebrates the responses to photic inputs are no longer so automatic. Very few motor responses are automatically elicited by variations in the optic array. However, as pointed out by Sperry (1952),

If there be any objectively demonstrable fact about perception that indicates the nature of the neural process involved, it is the following: in so far as an organism perceives a given object, it is prepared to respond with reference to it. This preparation-to-respond is absent in an organism that has failed to perceive. (p. 301)

Thus, consider the simple case where a person perceives a square of paper. The very act of perception means that the person now has a set of readinesses with which to respond to the paper. He or she may now point to it, outline it with a finger, localize it with reference to other objects, pick it up, walk around it, verbally describe it, or emit many other overt motor activities with reference to it. In a vertebrate that lacks the symbolic and linguistic capacities of the human being, this same principle is still operating. If the animal perceives the stimulus, it is able to approach it, avoid it, leap over it, pick it up with its mouth or paws, and so forth. In both the human and infrahuman situations, the organism that does not perceive the stimulus does not demonstrate any systematic motor responses toward it. Thus one might say that the presence of a pattern of efferent responses held in readiness, which is prepared to manifest itself as a pattern of motoric responses, indicates that the organism has perceived a given stimulus.

Because, in most of the simpler animals discussed here, the visual inputs are automatically used in guiding motoric outputs (generally in the form of locomotion, apprehension, or manipulation), one might suspect that this is the major adaptive function of the visual system. In more complex and elaborated animals, the visual guidance becomes mediated and modified by other factors such as internal states, other environmental stimuli, and behavioral strategies that have evolved as a function of the organism's past experience. However, the principal function of vision is still to guide the animal's efferent output. One testable implication of such a hypothesis is that the degree of sensory elaboration found in an organism should correlate with the amount of motoric activity in which the animal normally engages. Motile animals should have better sensory systems than nonmotile animals. It is difficult to assess the validity of this prediction across phyla because it is impossible to determine the basis on which to compare the mobility of an elephant with that of an amoeba. Perhaps the most valid comparisons are among animals within the same phyla. Consider a few examples to see if the expected correlation between motility and complexity of the visual sensory apparatus exists.

The segmented worms, or annalids, include a number of species varying in motility. In this phylum the degree of visualsensory specialization seems to vary directly with the motoric requirements and abilities of the animals. For instance, the sed- entary leeches are either totally lacking in eyes or display them in the most rudimentary forms. The more active but slowly moving earthworm has light-sensitive cells aggregated together to form simple eye spots. On the other hand the freely swimming polychaetes (sea worms such as nereis) show the most elaborate visual systems found in this group, with reasonably complex cupulate eyes.

In molluscs there is an even greater range of motility and sensory complexity. The bivalves, such as the clam, which spend most of their time lying on or below the sea floor, have very primitive light receptive organs, if any. The more motile but nonetheless generally quiescent forms, such as the scallop, begin to show aggregation of light-sensitive cells into eye spots. Snails and sea slugs are clearly more active and mobile than the scallop, though certainly not the most motile of animals. They show a more complex visual system, including an invaginated eye with a reasonably complex neural structure. The most active of the molluses are the cephalophods. These include the freeswimming octopus and squid, which have eyes that rival those of the vertebrates in developmental elaboration.

It is interesting to note that there are some instances in which the correlation between motoric activity and sensory elaboration manifests itself in the life history of the single animal. Thus in some species of mollusc, such as the common edible oyster mytilus, an active free-swimming larval stage precedes the sedentary adult pattern. During this motile stage, the animal displays cephalic, cupulate eyes that are reasonably well developed. During the stationary adult stage, however, these eyes become residual. This is actually not an atypical pattern for animals that have both an active and a passive stage of existence. It is found in the arthropods in a very similar form, as in the case of the ship barnacle lepas. In reverse temporal order, it is repeated in many insect species, wherein the relatively passive larval stage the insect is adorned by simple lateral eyes, whereas the more active adults display elaborate complex compound eyes.

It is possible to extend this mode of analysis to other phyla, and when one does so, it is clear that the pattern displayed above is the most typical. When one reaches the vertebrates, however, the range of both motility and elaboration of visual structure becomes more restricted; hence, the comparisons are more difficult to make. Nonetheless, the above examples make it clear that the degree of motor activity and complexity of the visual apparatus appear to be correlated. This would support a general notion that the essential, and perhaps a primary, function of vision is to guide movement. Movement must be controlled so as to allow the animal to achieve an optimal environmental situation, to avoid obstacles, or to pursue prey and avoid predators. In this sense one is led to expect complex visual organs to be found primarily in actively moving animals, because they are not needed by more sedentary species.

If these comparative data may be taken as providing some basis for concluding that the visual system and motor system are interrelated, what the nature of this relationship is and what consequences this may have on conscious visual perception, if any, must be asked. The hint as to the possible relationship comes from a consideration of the nature of linkages between the perceptual and motor activities. In the most primitive organisms, such as amoeba, the effect of light is directly on the protoplasm itself. The resultant alterations in its viscosity affect 
the direction of movement in such animals. The first evidence of cellular specialization is found in porifera. Here the arrangement is quite simple, with sensory cells directly adjacent to motoric cells. As the complexity of organisms increases, moving up the phylogenetic scale, neural elements are interpolated between the afferent input and efferent output units. In the complex vertebrates, there are many neural pathways that do not appear to go directly to motoric units. Thus, in the more complex species, there are numerous pathways to nonmotoric portions of the cortex. It looks as if the information that was initially extracted for motoric guidance is now being made available for "other purposes." One might guess that conscious perceptual experience is a direct consequence of these "other purposes." This would suggest that conscious perception may be directly affected not so much by the actual motoric outputs but by the process of information extraction that has been keyed to the needs of the motoric system. In turn, this would lead one to expect that information which is unnecessary to guide movement would not be readily available, because the system is basically not set up to extract such extraneous data from the incoming stimulus array. Furthermore, this might suggest that because visual information extraction is based on the requirements for motor guidance, the entire process might be rather selective as to the parameters it considers important.

\section{A Research Strategy}

In the preceding section a possible mechanism by which visual perception might be affected by efferent considerations was derived. An interesting hypothesis can be generated from such speculations. Suppose a set of conditions could be found under which the information extracted for motor guidance is biased in some systematic fashion. If it is correct to assume that the bulk of the information made available to consciousness is simply passed on to higher centers after it has been prefiltered and preselected to guide motor outputs, the resultant percept ought to also be systematically biased. Put in more operational terms, if the information used to guide the motoric response to a stimulus array differs from an accurate reflection of the physical characteristics of the stimulus array, this should manifest itself in a corresponding perceptual distortion or illusion. The extracted information is used to evoke a set of efferent patterns that are now held in readiness. These efferent readinesses, in turn, serve as the foundation for the conscious percept.

Up to now the research strategy used to investigate the effects of efferent readiness on perception has been the same one that would be used to investigate the effects of overt motoric involvement on perception. The rationale has been that because the best index of the eye movement held in readiness is the actual eye movement emitted, one need only measure the eye movement patterns across illusion configurations and look for systematic eye movement biases that may be correlated with the percept. As an example, this was the pattern of reasoning that guided some research by Coren, Bradley, Hoenig, and Girgus (1975). This research was based on an interesting illusory phenomenon that we observed a few years ago: If a spot of light is rotated in a circular path, in a totally darkened room, the apparent diameter of the circle seems to vary as a function of the speed of the target's movement. At slow speeds, subjects tend to accurately estimate the diameter of the circular path that the target is traversing; however, as the speed of rotation of the stimulus increases, the diameter of the circular path begins to apparently shrink. If the speed of rotation is further increased, the diameter of the circular path then seems to expand back to its normal size. This dynamic illusion of size caught our attention, because it seemed to be amenable to an explanation involving a motoric component. We decided to investigate it using the customary approach. We proceeded to measure eye movements as the subject attempted to follow the target in its circular path. The results were, in fact, consistent with an eye movement explanation. At slow speeds the subjects accurately tracked the circular movement of the stimulus, whereas at intermediate speeds subjects tended to track in a circular path with a reduced diameter. Furthermore, the perceptual underestimates of the diameter of the target track were highly correlated with the obtained diameter of the tracking eye movements. As the speed of the target increased beyond this intermediate level, more saccadic eye movements began to intrude. The proportion of saccadic eye movements to the outer limits of the target path increased and tended to correlate with the apparent expansion of the circle diameter outward toward veridicality. We followed the tradition of the motor theories of perception outlined above and concluded that the perceptual distortion was, at least in part, caused by the systematic mistracking of the stimulus. From the current vantage point, however, it seems likely that this conclusion is a methodological and philosophical trap. It may be useful to outline the nature of this trap.

A careful analysis of the present procedures constitutes a good starting point. Following the methodology typical of studies that have looked for motoric involvement in illusions in the past, our first studies used a particular illusory distortion. Next eye movements were measured during the occurrence of that perceptual distortion. The end result was the conclusion that eye movements are correlated, in both direction and magnitude, with the obtained illusory effect. Note that basically all that we had obtained at the end of that investigation was the simple correlation between the eye movement pattern and the percept. At the very least, it was presumptuous, if not foolhardy, to say anything about causal relationships with only this correlational data. It may be the case that eye movements do, in fact, provide some source of information that is eventually incorporated into the final percept, but it is also possible that the perceptual distortion itself is the primary factor causing the observed relationship. Thus, the perceived distortion may have elicited the erroneous eye movements as easily as the erroneous eye movements may have formed the basis for perceptual distortion. The sad fact is that the same argument could be used to explain away many of the results that seem to suggest that there is an interaction between eye movements and illusions.

As another example of the same problem, consider, for instance, the Mueller-Lyer illusion data discussed above. It may well be the case that, rather than the overly long eye movements' causing an overestimation of the shaft in the apparently longer segment, the perception of an apparently longer line serves as the basis for emitting the longer eye movement. The root of the problem is that the only item of data available is the correlation between the eye movement and the percept. Methodologically, and even more disturbing, is the fact that the eye movements 
are usually measured only after some perceptual distortion is found. It seems as if no one has ever been able to reverse the process, and to begin with the existence of a motoric bias, in a system such as eye movement, and from there demonstrate the existence of a perceptual distortion. Certainly, if there is any causal interaction, this ought to be possible. To state this even more forcefully, in all circumstances where the eye movements are distorted, illusory distortions ought to be predictable.

A possible research strategy can be reformulated on the basis of the above reasoning. To begin with, the normal procedure will be inverted. Suppose that some set of stimulus conditions known to produce a systematically biased set of eye movements can be found. If it is the case that eye movements or, more specifically, the information extracted to guide the eye movements interacts in the formation of the phenomenal percept, then one ought to be able to predict that these systematic biases in the eye movement patterns should be reflected as a set of biases (distortions or illusions) in the subjective percept. Next, increasing the set of constraints on the theoretical predictions, if we find any set of parameters that will systematically alter this biased eye movement pattern, we must also predict that these parameters will cause certain changes in the conscious perception of the array as well. Finally, a degree of generalizability should be added to the predictions. Thus, it must be predicted that any stimulus array that triggers this same systematically biased pattern of eye movements should also be accompanied by the same systematic distortion in the subject's conscious representation of the stimulus. Therefore, if there is a causal interaction between eye movements and perception, in any situation where the eye movements are biased, we should expect this to be accompanied by a predictable illusory distortion.

\section{Center of Gravity and Biased Eye Movements}

The above predictions have broad implications. However, they could be directly tested if some situation could be found where eye movements are systematically biased and that bias is susceptible itself to parametric manipulation. Fortunately, a simple stimulus situation exists where biased eye movements occur. A number of years ago a series of studies was conducted to assess the accuracy of voluntary eye movements (Coren \& Hoenig, 1972). These studies were inspired by an observation that when the pattern of eye movements that a subject emitted, expressed as the length of an initial saccade to a target, was recorded, certain arrays were found where these eye movements were systematically in error. Actually, the stimuli that elicit these biased eye movements are not very complex. Biased eye movement patterns begin to manifest themselves whenever there are nontarget stimuli in the vicinity of the target to which the subject is trying to direct his or her eye. Suppose, for instance, that the observer is viewing a central fixation point. Next, randomly, to either side of the fixation, a target is presented. When observers attempt to fixate this target, they tend to be fairly accurate. At this point an extraneous stimulus is introduced into the field. This extraneous stimulus is, for instance, a second target, clearly discriminable from the first in terms of either color or form and is located beyond the target. In this case, there is a tendency for the initial saccade to be longer; thus the eye tends to be pulled in the direction of the extraneous stimulus. Suppose, on the other hand, that the extraneous stimulus is placed between the initial fixation point and the target. Under these conditions there is a tendency for the saccade to be shorter. These findings were anticipated by Bruell and Albee (1955) and confirmed in a more recent report by Findlay (1981). From these observations, it was reasoned that the spatial information controlling the length of the saccade was not solely the locus of the target but rather some sort of estimate of the center of gravity of all of the stimuli in the immediate vicinity. This conclusion is also consistent with some observations that fixation patterns tend to be affected by the global pattern of stimuli present in the field (Kaufman \& Richards, 1969; Richards \& Kaufman, 1969).

The tendency to emit eye movements with a bias toward the center of gravity of stimuli in a particular region of the visual field, rather than directly toward the target of interest, is actually a sensible adaptation to the fact that the fovea is not punctate but effectively used as if it extended over several degrees. We may define a functional fovea as a $2^{\circ}-4^{\circ}$ circle around the center of the physiological fovea, where visual acuity is optimal. Any target falling within this region is seen with reasonable clarity. Because the function of the eye is to gather as much information as possible about the external environment, one practical adaptation would be to habitually direct eye movements toward regions in the visual field that contain the most visual stimuli and, hence, the most information. This bias toward the acquisition of the maximal amount of information possible with each fixation might, at times, result in saccadic eye movements that are not guided solely by the locus of the target the observer is ostensibly setting out to investigate but would still be quite adaptive.

As an example of the operation of this instrumental bias observable in eye movements, consider Figure $2 \mathrm{~A}$, in which the functional fovea, that is to say the region around the fovea in which high visual acuity is obtained, is depicted as a circle. Let us suppose that the eye is initially fixated on some point that is indicated by the letter $F$. First suppose that the individual wishes to observe some target stimulus $(T)$. It seems quite obvious that a saccadic eye movement would be programmed to center the image of the stimulus $T$ on the fovea. Next, the array is altered slightly so that an additional (extraneous) stimulus appears, here represented by the letter $X$ near the target $T$. Given the fact that the functional fovea is relatively broad, only a slight increase in the length of the saccade would be needed to image both stimuli on this region, as is shown in Figure 2B. This means, of course, that clear vision of both the target $T$ and the extraneous stimulus $X$ can now be obtained by the observer. Suppose that the extraneous stimulus $X$ is moved further away, as in Figure 2C. Now, if the observer lengthens the saccade a bit more, he or she can still place the image of both targets on the fovea. Notice, however, that there is a range beyond which lengthening the saccade becomes nonfunctional, as in the situation shown as Figure 2D. Here, the extraneous stimulus is a sizable distance from the target toward which the initial visual attention was to be directed. If the saccade were made long enough to bring $X$ onto the functional fovea, $T$ would no longer be adequately centered in clear vision. Because $T$ was the original target of interest, toward which the eye movement was to be directed rather than losing accurate registration of $T$, the strat- 


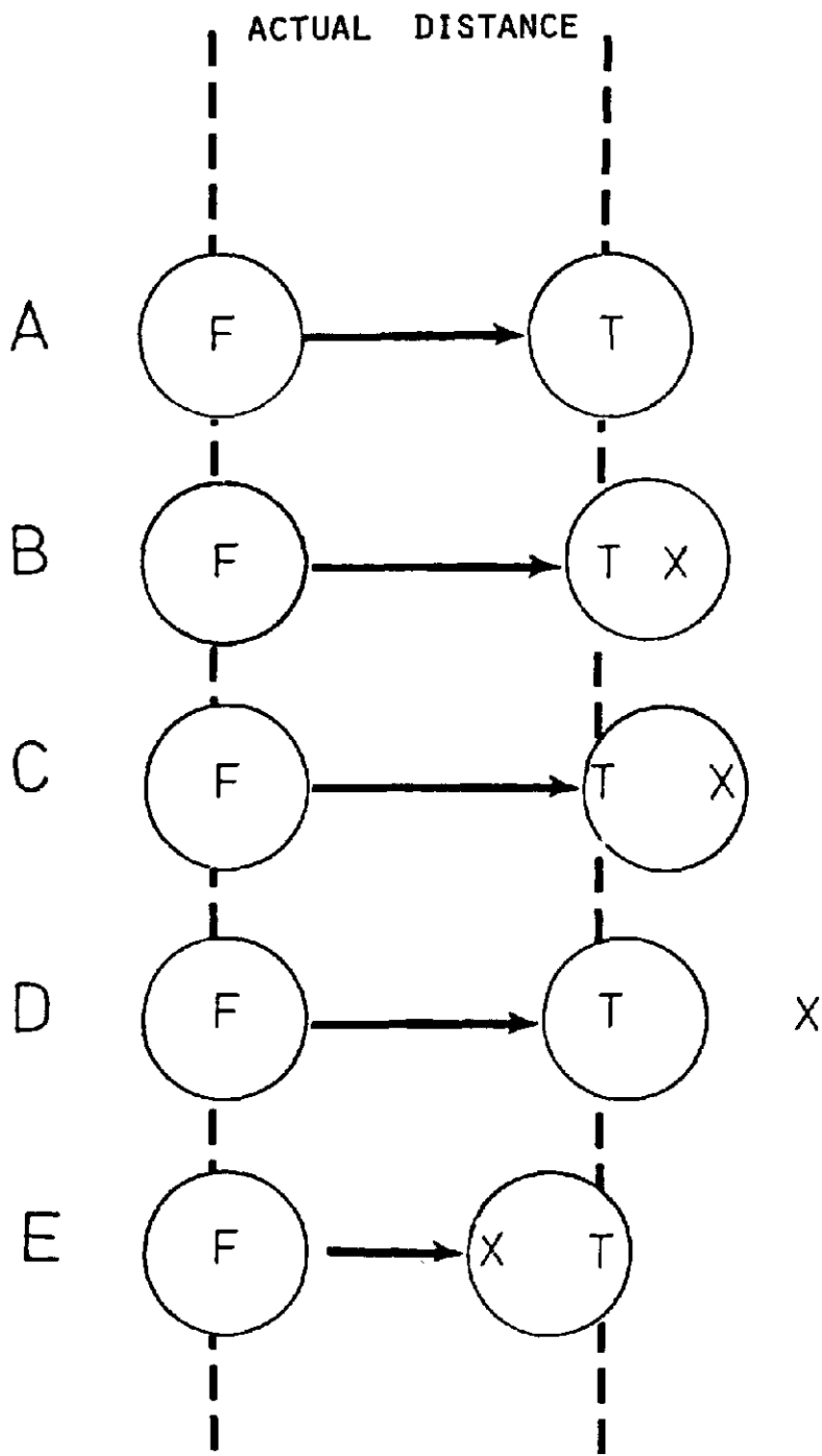

Figure 2. A: Unbiased saccade length from a fixation point $(F)$ to a target $(T)$. B-D: Alterations in saccade length for an extraneous stimulus $(X)$ placed beyond the target. E: Effect of an extraneous stimulus between the fixation and the target.

egy of lengthening the initial saccade to pick up information from nearby extraneous stimuli would most likely be abandoned in this situation. To summarize these speculations, it is predicted that as the distance between the target and the extraneous stimulus increases, the saccade will lengthen up to a point, beyond which it will begin to return to its unbiased length.

Of course, the converse effect occurs if the extraneous stimulus is placed between the fixation point $F$ and the target $T$ rather than beyond it. In such a case, the saccade could be slightly shortened in order to pick up additional information in the vicinity of the target. This effect is shown in Figure 2E, where the saccade length has been shortened in order to capture both the target $(T)$ and the extraneous stimulus $(X)$, which is now located nearer to the fixation point than is the target.

The resultant pattern of lengthenings and foreshortenings which would be predicted, as a function of locating the extraneous stimuli in various positions around the target, is somewhat like that shown in idealized form as Figure 3. In general, the observed eye movement patterns did follow such a pattern (Coren \& Hoenig, 1972). Typically, the initial saccade length was longer when extraneous stimuli were added beyond the target and was shorter when stimuli were present between the target and the initial fixation point. Similar patterns may be deduced from other observations in the literature (Bruell \& Albee, 1955; Findlay, 1980, 1981; Levy-Schoen, 1974; Levy-Schoen \& Rigaut-Renard, 1979).

These results seem to be rather robust. They are not due to poor discriminability between the target and the extraneous stimuli, because care had been taken to make sure that the targets and the extraneous stimuli were clearly discriminable from the target by variations in color or form. Subjects were well briefed in advance as to the possible presence of extraneous stimuli and were instructed to be as accurate in their eye movements as they possibly could. [Corroboration of this comes from other studies, where observers were asked to fixate the target in order to ascertain the orientation of a fine grating of lines (Coren \& Hoenig, 1972) or a gap in a figure (Findlay, 1981).] To perform these tasks well, the subject had to center the target in the foveal region where acuity was best; nevertheless, the same systematic eye movement biases in the direction of extraneous stimuli occurred. Thus it appears that the eye movements

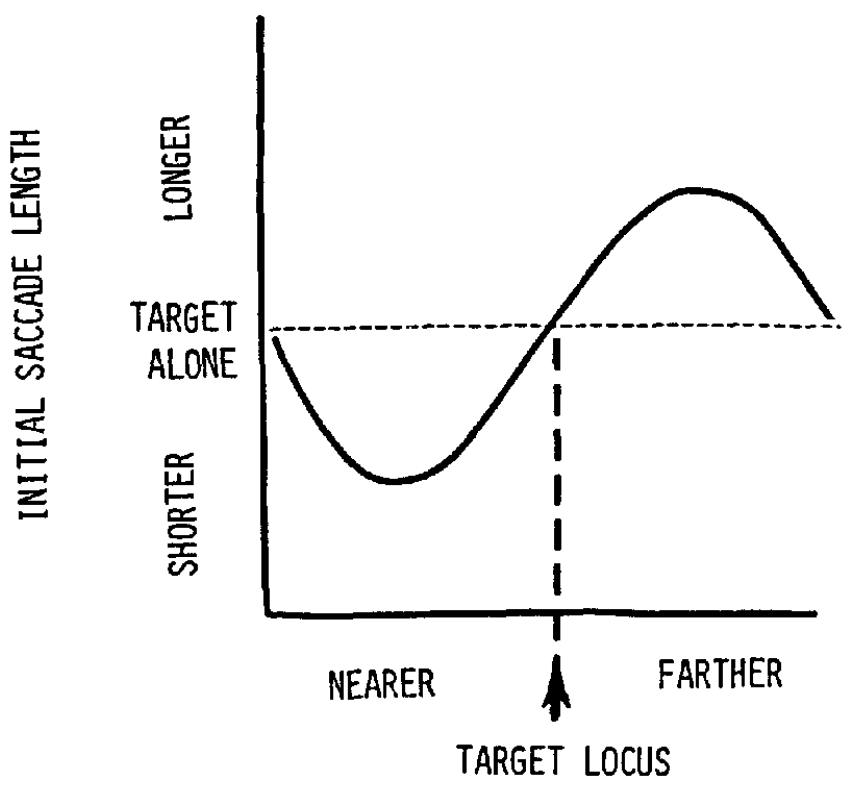

EXTRANEOUS STIMULUS POSITION

Figure 3. An idealized version of the increase and decrease in initial saccade length in the presence of an extraneous stimulus placed beyond the target (as in Figure 2B) or closer to the fixation than the target (as in Figure 2E) derived from data from Coren and Hoenig (1972). 
were lengthened or shortened on the basis of considerations not amenable to voluntary conscious control. The eye movements themselves seem to be controlled simply by the information pickup requirements that directed the eye toward the center of gravity of stimuli in a given locus in the vicinity of the target stimulus.

The importance of these findings is that they provide a simple set of stimulus conditions where patterns of eye movements emitted over a stimulus array can be systematically varied and where they do not simply reproduce the veridical location of targets in the visual field. Innumerable stimulus configurations can be created, which should produce a variety of biased eye movement patterns, by merely introducing extraneous stimuli and altering their location relative to the target elements. As long as the intended target can fall in the area of clear vision on the functional fovea, the eye movement will be biased toward the center of gravity of other stimuli in the immediate surround. As the center of gravity moves outward, or inward, the length of the saccade emitted will correspondingly lengthen or shorten.

Returning to the theoretical issue of the relationship between eye movement efference and the visual percept, consider the implications of these findings. A situation has been isolated in which the eye movements are systematically longer or shorter than those that would be emitted to a target presented in isolation. Suppose, as any strict efferent theory must maintain, that the information available to the sensorium (or higher conscious centers) is predominantly composed of the information that has been extracted to guide motor output. If a set of configurations or conditions is then isolated in which the motoric output is systematically biased, it ought to be the case that the perception of such configurations ought to also be systematically biased in a similar manner. To place the prediction within the context of the data discussed above, this would indicate that the perception of the location of stimuli, or extents bounded by stimuli, that appear in the presence of extraneous stimuli ought to be biased. Specifically, they ought to be biased in directions predictable from the location of the center of gravity in a specific region of the visual field. Furthermore, in this form of an efferent theory, we do not require the actual eye movement to be emitted. Rather, the bias arises from the computation of the saccade that would be made and, hence, is held in readiness, rather than the saccade actually emitted.

\section{Demonstration 1: Primary Center of Gravity Illusion}

In the preceding section it was suggested that the incoming afferent stimulation and the outgoing motor innervation form a single continuous nerve process. Conscious visual perception is simply one product of the preparation for motoric response, in much the same manner that a preparatory tightening of some muscle groups might be. A situation where systematically biased eye movements can be elicited has also been described. To the extent that the analysis is correct, the same conditions that produce these deviations in the eye movement patterns also should produce a predictable corresponding perceptual distortion. In effect, it is predicted that a hitherto unreported set of illusions can be created by using elements that produce systematically biased eye movements, namely, targets and nearby extraneous stimuli.
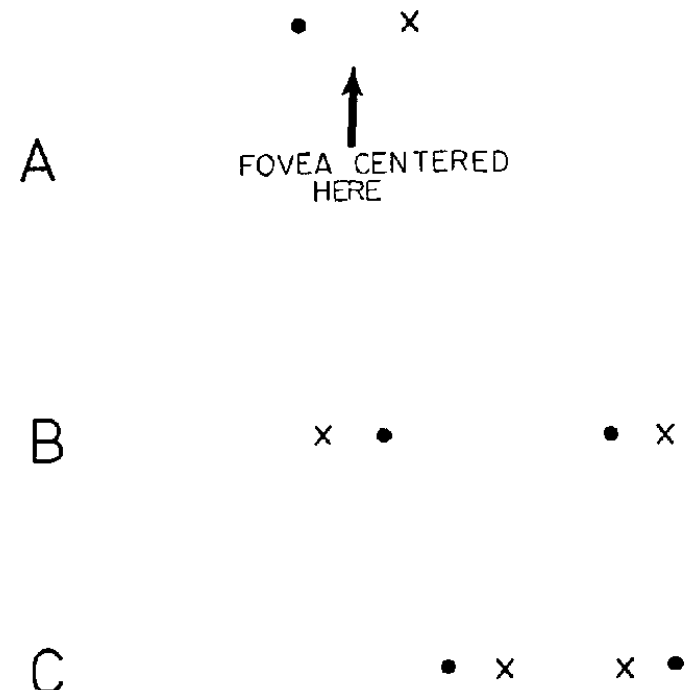

Figure 4. A: A target stimulus (dot) and an extraneous stimulus $(X)$ and the hypothesized position of the the foveal center between them. $B$ and C: Primary center of gravity illusion created from this basic unit (B representing an overestimation of the distance between the dots, relative to the underestimated portion C).

This discussion will begin with the simplest stimulus unit to create what might be called the primary center of gravity illusion. Figure 4 shows the basic component that can be used to create a series of stimuli that should, according to the theoretical analyses above, result in perceptual misjudgment. This component consists simply of a target (here a dot) and a clearly discriminable extraneous stimulus (here an $X$ ). If the observer were asked to fixate the dot, on the basis of the eye movement data that have been considered thus far, the fovea should be centered, not symmetrically around the dot but displaced toward the extraneous stimulus. In other words, the fovea should be centered at approximately the position indicated by the arrow in Figure 4A. Of course, given only this basic unit, no extent or direction that should be misjudged has been defined. However, it is easy to concatenate these basic units (composed of a target and an extraneous stimulus) to produce an array that might produce a measurable perceptual distortion (see Figures $4 \mathrm{~B}$ and 4C). Notice that in Figure 4B, two dots define a linear horizontal extent. In this array the extraneous stimuli are placed in such a position that if the observer were asked to look at the leftmost dot and then to shift to the rightmost dot, the resultant saccade should be longer than if the observer were asked to look at the leftmost dot and then to shift his or her gaze to the rightmost dot in Figure 4C. This occurs because the length of the saccade is biased toward the center of gravity of the stimulus complexes that serve to define the extent, as illustrated in Figure 4A. This effect is directly predictable from the Coren and Hoenig (1972) data depicted earlier as Figure 3. If it is the case that the data extracted to guide such eye movements interact with the final percept, one might expect these different patterns of eye movements to result in the apparent overestimation of the distance between the dots in the upper configuration relative to the lower. The presence of the predicted distortion should be apparent to the reader who attempts to compare these extents in Figures 4B 
and 4C. Most observers do see the expected distortion in the array, with the upper extent between the two dots appearing longer than the lower extent between the two dots.

Even though the expected illusion appears to be present from casual viewing of Figure 4, a series of more formal measurements were taken using a sample of 20 student volunteers. They were presented with figures equivalent to those in Figures 4B and $4 \mathrm{C}$. The dots were separated by $8 \mathrm{~cm}$, and the extraneous $X$ was $1 \mathrm{~cm}$ from the dot. The viewing distance was $40 \mathrm{~cm}$. Subjects were asked to adjust a variable extent so that it appeared to be equal to the distance between the two dots in each of the arrays. As the theory predicted, subjects overestimated the distance between the dots in the upper figure by $4.1 \mathrm{~mm}$ and underestimated the distance in the lower figure by $3.2 \mathrm{~mm}$. Both of these differences are statistically reliable, $t(19)=4.17$, $p<.01$, and $t(19)=3.92, p<.01$.

The discovery of this previously unknown illusion of extent, using only predictions made on the basis of eye movement data, is encouraging. However, the mere existence of the illusory distortion is not enough. It must also be demonstrated that the magnitude of this illusion can be parametrically varied by the same factors that vary the observed eye movement biases.

\section{Demonstration 2: Parametric Variation of the Illusion}

In its strongest form, the efferent theory being advocated suggests that anything varying the efferent readinesses, or the information extracted to guide potential eye movements, should also vary the perception. Although the basic form of the center of gravity bias of the eye movements allowed for the prediction of the existence of an illusion, it is known that this bias is subject to parametric variation. On the basis of the data schematically summarized in Figure 3, it is known that the eye movement biases will vary as a function of the distance between the target and the extraneous stimulus. On the basis of that variation, it can be predicted that the magnitude of the illusion will similarly change. Specifically, the illusion magnitude should increase as the distance is increased between the extraneous stimulus and the target. This increase, mimicking the eye movement pattern, will only continue up to some intermediate distance. It is known that in the eye movement pattern, when it is no longer functional to continue lengthening the saccade because too much acuity would be lost, this strategy is abandoned, as illustrated in Figure 2. Extrapolating to the perceptual situation, this implies that when the extraneous stimulus is far enough away from the target, the distortion should be expected to then decrease in magnitude.

To test these predictions, the configuration shown in Figure 5A was created. With an adjustable tongue and groove apparatus, 11 subjects were required to set the distance between the center and the right-hand dots so that they appeared to be equal to the distance between the center and the left-hand dots. Notice that the left side of the configuration contains the basic distortion producing stimulus, namely, a target (dot) and an extraneous stimulus $(X)$ beyond it. On the basis of the data from the preceding experiment and the eye movement data, it was expected that the extent on the left side would be overestimated relative to the extent on the right.

The extraneous stimulus could be located at one of seven dis-
EXTRANEOUS STIMULUS
DISTANCE

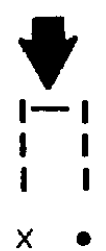

ADJUSTABLE LENGTH

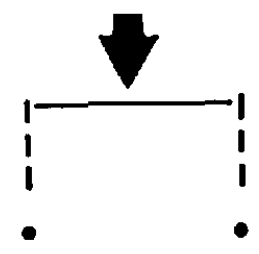

A

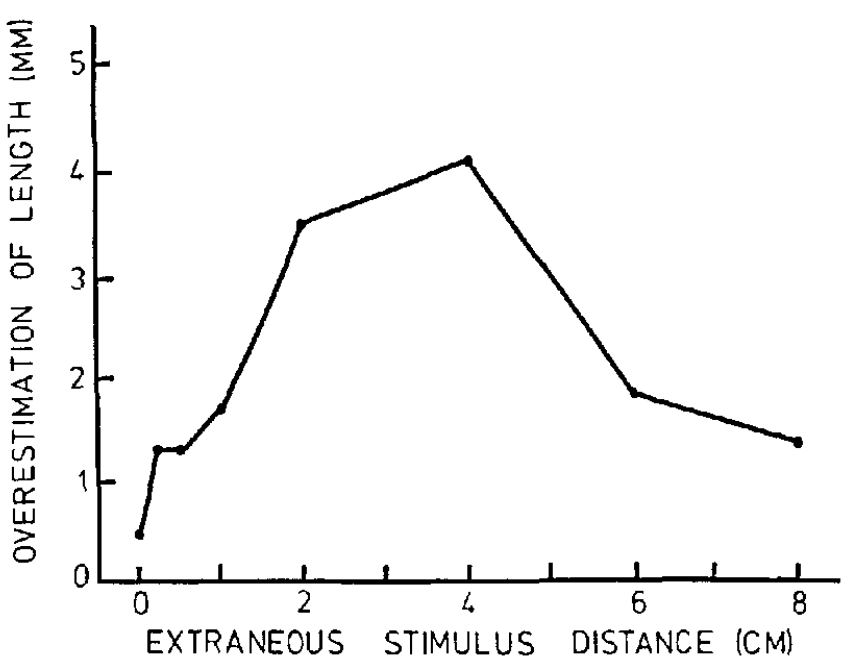

Figure 5. A: Stimulus used in Experiment 2 (the distance between the pair of dots on the right is adjustable; the distance between the left pair of dots is overestimated as a function of the distance of the extraneous stimulus $[X])$. B: Systematic change in the degree of overestimation as a function of varying the position of the extraneous stimulus.

tances from the left-hand dot. These distances were $0.25 \mathrm{~cm}$, $0.5 \mathrm{~cm}, 1 \mathrm{~cm}, 2 \mathrm{~cm}, 4 \mathrm{~cm}, 6 \mathrm{~cm}$, or $8 \mathrm{~cm}$ beyond the test stimulus. Of course, a control configuration in which there was no extraneous stimulus was also used to serve as a baseline. The obtained results are shown graphically in Figure 5B. From the shape of the obtained curve, it is obvious that the distance between the extraneous stimulus and the target stimulus does affect the magnitude of the illusion. The illusion increases in magnitude up to a maximum and then decreases as the distance between the extraneous stimulus and the target increases. This finding exactly parallels the eye movement data schematically presented in Figure 3. The effect of extraneous stimulus distance was statistically significant, $F(7,77)=2.38, p<.05$. Equally as important is the fact that a significant quadratic trend was obtained, as predicted by the eye movement pattern, $F(1,77)=5.53, p<.05$.

The theoretical significance of this experiment is that it indicates that one can predict a set of perceptual distortions armed 
with only a knowledge of some simple biases and the pattern of eye movements emitted across an array. Notice that the eye movement data first suggested a new form of illusion, and then suggested the parametric variation of the illusory effect as stimulus parameters were varied.

\section{Demonstration 3: Relationship to Classical Illusion Figures}

Although this is a newly discovered illusion configuration, the principles that led to its creation do allow for a number of statements and predictions about some of the classical visual-geometric illusion figures. Many of the more familiar visual-geometric illusions may be analyzed into component clusters, which also consist of target elements or loci, and extraneous stimuli, which serve as the illusion-producing elements. For example, consider the overestimated segment of the Mueller-Lyer figure (the upper portion of Figure 6A). If the target loci are considered to be the vertices defining the horizontal extent to be judged, it is easy to see how the outwardly turned wings in this figure might serve as extraneous stimuli that shift the center of gravity outward from the vertex into the body of the angle. The result is a stimulus array that on the basis of the predictions from eye movement data would have one expect an overly long saccade and a corresponding overestimation of the extent between the pairs of outwardly turned wings. In effect, the Mueller-Lyer configuration is theoretically equivalent to the overestimated segment of the center of gravity illusion (Figure 4B). Conversely, the inwardly turned wings will tend to shift the center of gravity inward, thus producing conditions for underestimation of length (lower segment of Figure 6A). Again, at the theoretical level, this stimulus is equivalent to Figure $4 \mathrm{C}$. This classical configuration can be stripped to its theoretical essence, demarcating only the location of vertices (targets) with dots and replacing the wing tips (extraneous stimuli) with dots, as in Figure 6B. This minimal, or dot, variation of the Mueller-Lyer illusion should, according to the analysis thus far, still produce the classical overestimation with the outwardly turned elements and underestimation with the inwardly turned elements. As can be seen from Figure 6B, the classical illusion is still obtained. Coren (1970) has verified that the illusion still exists in this form, although it is somewhat reduced in magnitude, suggesting that other factors may serve to augment the basic effect. It is possible to remove most of the line segments from a number of
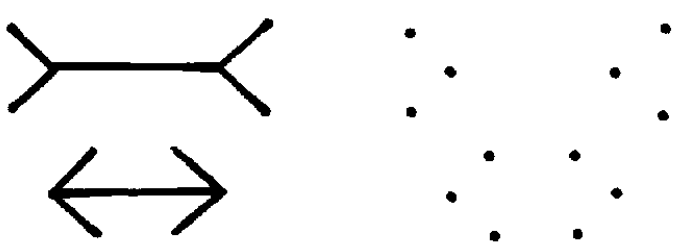

A

Figure 6. A: Standard form of the Mueller-Lyer illusion. B: a dot form of the Mueller-Lyer illusion, keeping only dots for the target stimuli (formerly the vertices) and for the extraneous stimuli (formerly the wing tips).

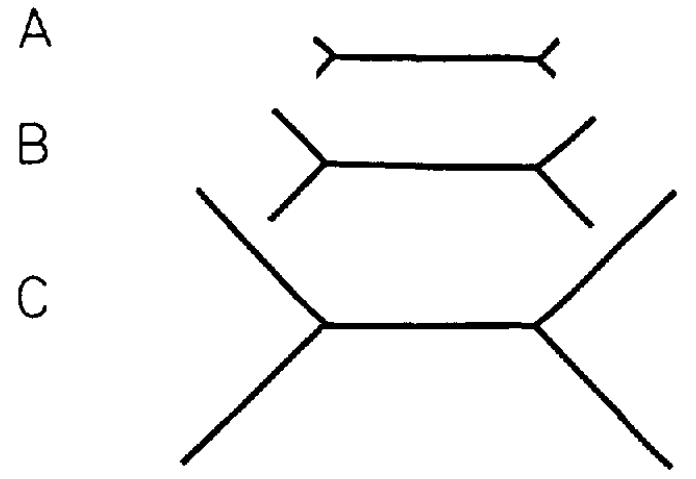

Figure 7. Demonstration of the effect of wing length on the magnitude of the Mueller-Lyer illusion. (As the wing length increases from A to B, the overestimation increases, but the size of the distortion is weakened if the wings are too long, as in C.)

classical illusion figures, replacing the (target) vertices and the (extraneous) line ends with dots and still to obtain the usually expected illusory distortions (Coren, 1970; White, 1972). These findings are consistent with an analysis based on shifts in the eye movement readinesses as one shifts the center of gravity of local clusters of stimuli in these classical illusions.

The preceding paragraphs indicated that some of the same modes of analysis that can explain the center of gravity illusion can also be used to explain aspects of other visual-geometric illusions. To the extent that this is true, one should then expect that a configuration like the Mueller-Lyer also ought to be subject to the same parametric variations that alter the magnitude of the center of gravity illusion. For instance, in the previous section it was found that the distance between the target stimulus and the extraneous stimulus affects the magnitude of the center of gravity illusion. Within the context of the MuellerLyer figure, as reanalyzed here, it is suggested that the magnitude of the illusion should vary as a function of the distance between the wing tip and the vertex, or more simply as a function of varying the wing length on the figure. Thus, one should be able to predict that as the wing length increases, the illusion magnitude will increase up to some maximum and then decrease in exactly the same pattern observed in the previous experiment. A simple demonstration is shown in Figure 7, which suggests that such may be the case. Notice that as the wing length is lengthened from Figure 7A to Figure 7B, there is a noticeable increase in the apparent length of the horizontal shaft. As the wing length becomes significantly longer, as in Figure $7 \mathrm{C}$, the magnitude of the overestimation begins to diminish. Thus the greatest overestimation is found for the wings of intermediate length.

More formal measurement of the effect of wing length variation was obtained using a sample of eight observers. The stimuli involved only the apparently longer half of the Mueller-Lyer figure, with a shaft length of $6 \mathrm{~cm}$ and an angle between the wings of $90^{\circ}$. The wing length was varied and could be $0.5 \mathrm{~cm}$, $1 \mathrm{~cm}, 2 \mathrm{~cm}, 4 \mathrm{~cm}$, or $8 \mathrm{~cm}$ in length. Estimates of the apparent length of the shaft (the distance between the two vertices) were made using a variable line length. Subjects adjusted it until it 


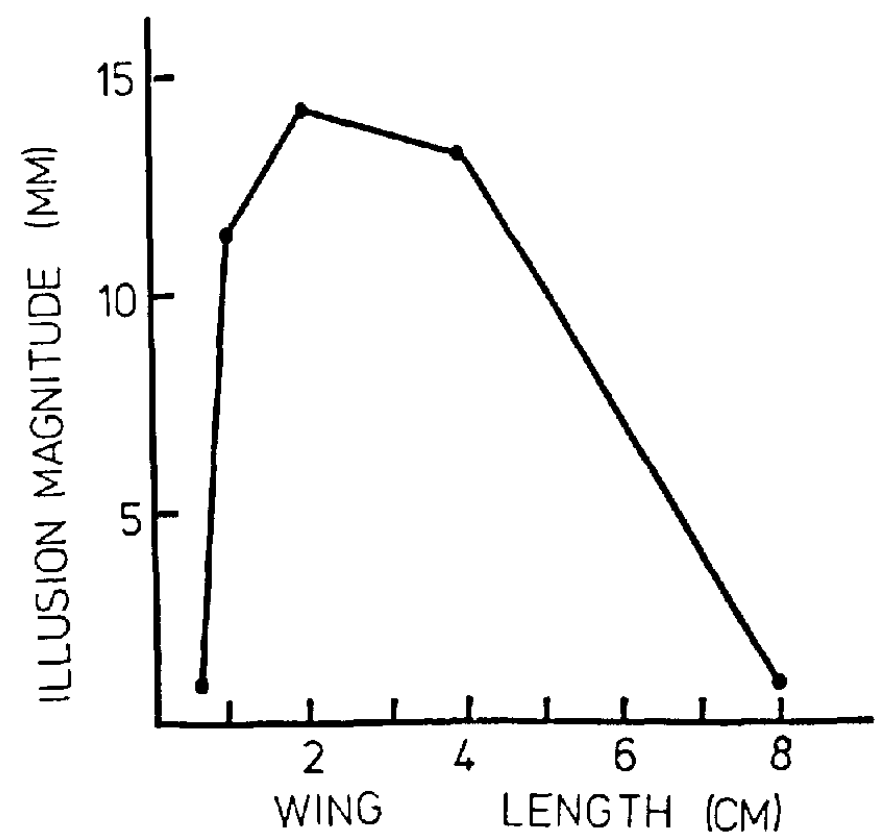

Figure 8. Experimental data indicating the change in the magnitude of the Mueller-Lyer illusion as a function of changes in wing length.

appeared to be equal to the length of the shaft. The results of these measurements are shown in Figure 8.

As can be seen from the figure, illusion magnitude varied as the wing length increased, producing an inverted U-shaped curve. This pattern of results is very similar to that obtained for variations of the center of gravity illusion (Figure 5) and is consistent with the suggestion that the wing tips serve as the extraneous stimulus and the vertex as the target in this figure. The effect of the wing length is statistically significant, $F(4$, $34)=4.31, p<.01$. The increased illusion magnitude for the intermediate wing lengths is further confirmed by the existence of a significant quadratic component, $F(1,34)=8.14, p<.01$. This is an important extension of the analysis, because it represents a prediction made, originally on the basis of the eye movement data, confirmed in the primary center of gravity illusion and now extended to a standard, well-known illusion configuration.

\section{Demonstration 4: Angle Changes and Center of Gravity}

In the preceding discussion the contribution of the wings to the Mueller-Lyer illusion was redefined. On the basis of the theoretical considerations thus far, the presence of angles at the line ends is not the critical illusion-producing factor; rather, it is the relative horizontal distance between the wing tips and the line end. Once the issue has been redefined in this way, a long-standing conflict in the illusion literature can be addressed. There have been many reports suggesting that the magnitude of the Mueller-Lyer illusion varies as a function of its angle. Unfortunately, the specific pattern of variation is somewhat ambiguous. Several investigators have reported that as the angle between the wings becomes smaller, the magnitude of the illusion increases monotonically (Dewar, 1967; Heymans, 1896; Lewis, 1909).
There are, however, some discrepant reports. Some investigators have reported that the magnitude of the illusion increases up to some maximum, usually at an intermediate angle size such as $45^{\circ}$ or $60^{\circ}$ and then decreases as the angle becomes even smaller (Auerbach 1894; Brentano, 1892; Nakagawa, 1958; Restle \& Decker, 1977). A simple resolution to these apparent contradictions can be offered, based on a reanalysis of parametric variations in the distance between the target (vertex) and the extraneous stimulus (wing tip) distance, which occurs with changes in wing angles.

Consider Figure 9A, which depicts two variants of the overestimated segment of the Mueller-Lyer configuration. In this figure the horizontal distance between the vertex and the wing tip for both an acute and a more obtuse angle have been marked off. It is clear that with the wider angle the horizontal distance between the target vertex and the extraneous wing tip is less than when the angle is more acute. This means that the center of gravity (roughly at the arrows) is relatively more displaced in the acute angle. This means that the magnitude of the overestimation of the illusion should be affected by changes in the angle of the wings, a result that has been reported many times before for this figure (see Coren \& Girgus, 1978a).

An interesting and novel prediction comes out of the above analysis, namely, that there should be a predictable interaction between wing length and angie size. If the wing is relatively long, then as the angle is made more acute there is a greater change in the horizontal distance between the wing tip and the vertex than if the wing is short. This difference can be seen in Figure 9 B. Simply put, there will be a greater horizontal variation of the center of gravity for long wings then for short. In the present context, variations in the center of gravity result in variations in the illusion magnitude, with an increase up to some point, followed by a decrease. This implies that if wings of the MuellerLyer illusion are longer, so that there is a large horizontal variation in the center of gravity, then as the angle is made more acute the inverted $U$-shaped function similar to that in Figure 5 should be obtained. If, on the other hand, the wing is shorter, variations in the angle merely increase the horizontal distance
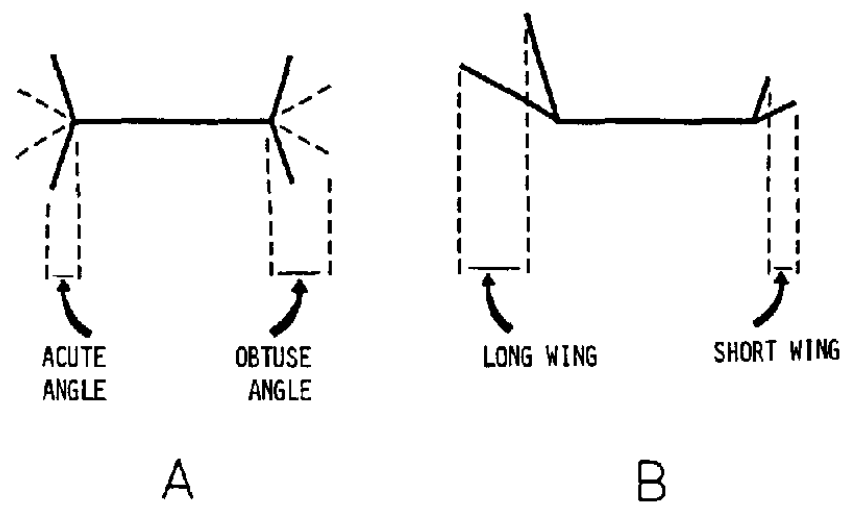

Figure 9. A: Demonstration of how variations in angle size translate into variations in the horizontal distance between the vertex and the wing tip of the Mueller-Lyer illusion. B: Demonstration that for an equivalent change in angle size, there is a greater variation in the horizontal distance (vertex to wing tip) for longer wing lengths. 


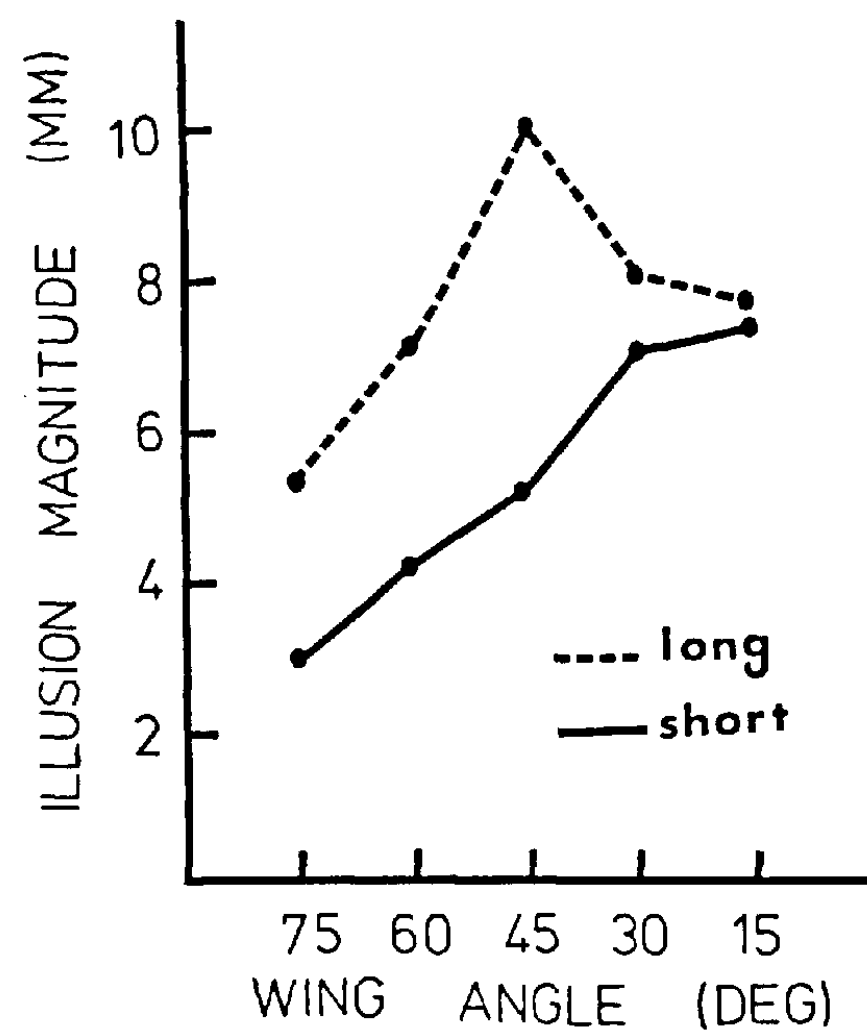

Figure 10. Variations in the magnitude of the Mueller-Lyer illusion as a function of changes in wing angle, for two different wing lengths.

toward the maximum without ever reaching the point where the center of gravity is shifted sufficiently far out for the strategy of lengthening eye movements to be abandoned. In other words, for a short wing tip there should only be an increase in illusion as the angle becomes more acute.

A group of 12 observers were tested for the predicted interaction between wing length and angle. The apparently longer half of the Mueller-Lyer figure was again used. The shaft length was $8 \mathrm{~cm}$, and the wing length was either $2 \mathrm{~cm}$ or $6 \mathrm{~cm}$. The angle between the wings and the horizontal line could be $75^{\circ}, 60^{\circ}, 45^{\circ}$, $30^{\circ}$, or $15^{\circ}$. Each of the observers adjusted a variable line length until it matched the apparent length of the horizontal extent of each configuration.

Figure 10 shows the results of this experiment. To begin with, notice that as the angle between the wings is made more acute there is a gradual increase in the overestimation of the figure when the wings are short. This results in a significant linear trend toward increasing illusion magnitude with more acute angles, $F(1,54)=8.63, p<.01$. For the longer wings, however, where the horizontal distance between the line and the wing tip would be expected to vary over a considerably longer distance as the angle becomes more acute, the results are quite different. Here the inverted U-shaped function, as predicted by the analyses in the previous two experiments, is obtained. This is confirmed by the presence of a significant quadratic component, $F(1,54)=9.60, p<.01$.

This finding helps to resolve the discrepancies between re- ports of the effect of angle size on the magnitude of the MuellerLyer illusion by pointing out that the wing length may have been a moderator variable that influenced the final pattern of results. More importantly, for the theory under discussion, it shows that some fairly complex patterns of results are predictable solely on the basis of a reanalysis of the array into components that allow for predicting shifts in the center of gravity in local regions of the figure and for assessing the effects that these shifts might have on the patterns of eye movements held in readiness.

\section{Demonstration 5: An Illusion of Location}

This discussion has been directed toward the demonstration that simple knowledge of eye movement patterns can be used to predict a number of systematic variations in some new and classical illusions on the basis of the theoretical presumption that the pattern of eye movements held in readiness does in fact affect visual perception. This process can be extended and used to predict a different class of illusory effects involving not only the perception of extent but also of direction.

Consider Figure 11A. Predictions from eye movement data would indicate that if observers were asked to look at the end of the horizontal shaft, they would tend to have their eyes pulled in the direction of the wing tips, here rightward into the body of the angle on both ends of the shaft. This is, of course, because the wings simply serve as extraneous stimuli relative to the vertex of the angle. The centers of gravity (indicated roughly by the arrows), and hence the eye movement readinesses, should be biased too far to the right when looking at either end of the figure. If the eye movement tendencies are components that enter into the formation of the final percept, then an interesting illusory effect can be predicted. Although one would not expect the length of the shaft to be distorted, because the biases are both in the same direction, one would expect the end points, which define horizontal extent, and hence the entire figure, to be mislocalized in space. Simply put, the eye movement tendencies will be biased toward the right side (away from the direction in which the arrow is pointed). The entire figure should be perceptually located too far to the right. Notice that this then is an illusion pertaining to the mislocation of an object in space rather than a simple illusion of extent.

This predicted illusion of location could be measured in one of two ways. The simplest and perhaps most direct way is to ask observers to indicate the position of the line ends. They should mark the ends too far to the right, indicating an apparent shifting of the figure in space. Alternatively, observers could be asked to bisect the horizontal extent. When they attempt to do this, they will, of course, bisect the apparent figure. If the prediction is correct, the figure is apparently shifted toward the right; thus the bisector, indicating the midpoint of the line, should also be placed too far to the right.

Both of these procedures do, in fact, indicate that the figure appears to be displaced in space (see Figure 11B). The mark on the horizontal shaft accurately bisects the figure, yet it appears to be too far to the left. This is consistent with the figure's being apparently displaced to the right. Two registration marks, perpendicularly aligned with the vertices of the configuration, have been placed on the horizontal line below the figure. Notice that 


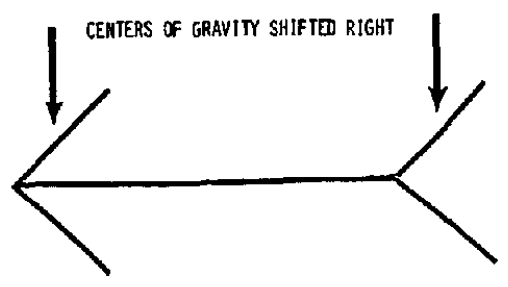

A

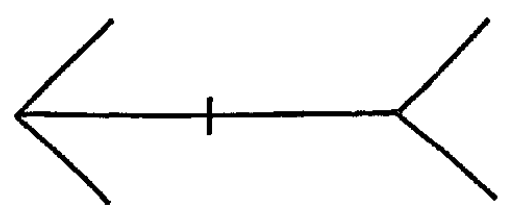

Figure 11. A: Center of gravity at both ends of the horizontal shaft is shifted in the same direction as indicated by the arrows. B: Resultant illusion of direction where the figure is seen as displaced too far to the right, relative to the hatch marks on the horizontal shaft, which actually indicate the location of the vertices; this same apparent displacement causes the mark bisecting the shaft to appear to be too far to the left.

they also appear to be too far to the left, again suggesting that the entire figure is apparently seen as displaced toward the right.

Even though the demonstration in Figure 11B seems convincing, a more formal experimental demonstration was conducted to verify these observations. Fifteen observers were tested on a configuration like that in Figure 11. The shaft was 8 $\mathrm{cm}$ long, the wings were $4 \mathrm{~cm}$ in length, and the angle between the wings was $90^{\circ}$. At $8 \mathrm{~cm}$ above and below the figure was a horizontal line parallel to the shaft of the illusion figure. Each observer made eight judgments, either placing a pencil mark at the apparent midpoint of the shaft or indicating the apparent location of the two end points of the line on the horizontal line beneath the figure. The orientation of the figures (either pointing toward the right or toward the left) was randomly alternated.

The results showed that the mean displacement of the apparent bisector of the shaft was, in fact, displaced away from the arrow head of the configuration. The average displacement was $3.6 \mathrm{~mm}$, which is statistically significant, $t(14)=3.11, p<.01$.
When the mean displacement of the figure is computed on the basis of the indication of the location of the vertices, again the figure is also found to be apparently displaced in a direction away from the arrowhead for an average of $2.3 \mathrm{~mm}$. This displacement is statistically significant, $t(14)=2.93, p<.025$. Because both of these displacements are in the same direction, it is assumed that they are both due to the apparent mislocation of the figure in space and to biases introduced by the efferent readinesses for eye movement. It is expected that for any given subject, the magnitude of the displacement of the bisector and the apparent displacement of the figure as measured by the location of the line ends should be correlated. In fact, they are quite highly correlated, $r=.62, p<.01$. These data taken together suggest that the predicted displacement of the figure in space is, in fact, occurring in this configuration.

This result is theoretically interesting because, in addition to verifying the predictions based on efferent readiness and the center of gravity considerations, it shows that the same principles that allowed one to predict aspects of the perception of visual extent also allow one to predict aspects of the perception of visual direction.

\section{Demonstration 6: Breaking the Correlational Loop}

That visual perception is affected by biases in the information extraction used to guide eye movements and to compute a set of efferent readinesses was the starting point in this analysis. Next, a simple stimulus situation was found in which eye movements were metrically different from those expected on the basis of accurate scanning of the stimulus array. Furthermore, some conditions under which the eye movement patterns could be systematically varied were specified. Then, from these eye movement patterns a series of hitherto unreported illusions and some systematic variations in the magnitudes of some classical illusions were predicted. Although the above performance may seem to strongly bolster the contention that there is an efferent component in the visual perception of extent and direction, a more dispassionate observer might argue that the basic methodological trap characterizing all former attempts to establish a motoric component in visual perception has not been avoided. The problem is that when one experimentally establishes that in a set of visual illusions the eye movement patterns mimic the distorted perceptual patterns in consciousness, all that has really been established is a simple correlation. There is no a priori way of determining whether the distorted eye movements cause the illusion distortion or whether it is the perceived illusion that elicits the erroneous eye movements.

It could be argued that this is a somewhat stronger position than that of most previous efforts, because the starting point was a series of eye movement biases in a situation where no perceptual distortions were formerly known to exist, and from these, perceptual distortions and their parametric variations were predicted. However, it could still be said that the correlation between the eye movements and the percept is the only item of data available, regardless of whether one starts from the percept and works toward the eye movements or takes the converse approach. Perhaps it is the case that any configuration producing biased eye movement patterns does so simply because any such configuration is already producing some form of percep- 
tual distortion. Nonveridical eye movements then simply become a sensitive measure of the existence of some visual illusions in the array.

Is it possible to resolve these ambiguities as to the direction of causality and thus break out of the correlational loop? Fortunately, a rather simple manipulation promises a way to do so. To start, note that most theories used to explain visual illusion are holistic in their orientation. They depend on the specific interrelationships among components in the array. The gestalt results in the final distortion, whether due to assimilation or contrast effects, or the triggering of inappropriate depth processing, and so forth (see Coren \& Girgus, 1978a; Robinson, 1972).

The pattern of theorizing employed here, based on the integration of efferent readinesses into the conscious percept, does not depend on the figure as a whole but rather on local configurational properties that affect the eye movement readinesses. Whereas virtually all other current theories of visual illusions would maintain that regardless of where on the array the observer's eye is dwelling the obtained pattern of distortion will remain the same, it might well be predicted here that as the eye moves around a stimulus array, because the relationship between the pattern of extraneous stimuli in a given locale may alter, the magnitude of the various illusory distortions may also change. If this is the case, it may give a means of separating the eye movement control aspects from the configurational aspects of the stimulus.

To understand how the efferent readiness patterns guiding eye movements may change, depending on where in the array the eye is dwelling, consider a simple example using a variant of the primary center of gravity illusion consisting of a target and an extraneous stimulus. Consider Figure 12A, where the eye is resting on a starting point (dot), indicated by the arrow. The broken line indicates the primary eye movement length that one would expect if the eye were to move to another target dot. Of course, as discussed, the eye movement will be longer than this if there is an extraneous stimulus $(X)$, as in Figure 12B. Suppose that the viewing situation is slightly different and the eye is already accurately centered on a target next to an extraneous stimulus, as shown in Figure 12C. In this case, although the configurational properties in the stimulus are the same, the eye movement readinesses are computed on the basis of a movement to an isolated target rather than a target in the vicinity of an extraneous stimulus. Therefore, it seems reasonable to expect that the eye movement readiness would produce a saccade that more accurately reflects the actual angular extent shown in Figure $12 \mathrm{C}$ than it would in Figure 12B. To the extent that the initial set of efferent readinesses evoked by the stimulus are reflected in the conscious percept, it is suggested that despite the fact that the stimulus arrays are the same (only laterally mirror imaged here for convenience) in the first situation, one would expect an illusory overestimation of the distance between the two dots, whereas in the second case, one would expect that the distortion would be greatly attenuated or perhaps eliminated. According to an efferent theory, where part of the perception of the size of the span between the dots depends on the eye movements held in readiness, the presence or absence of the illusory distortion will depend, in part, on the location of the eye on the stimulus array because this can alter the efferent readinesses evoked by

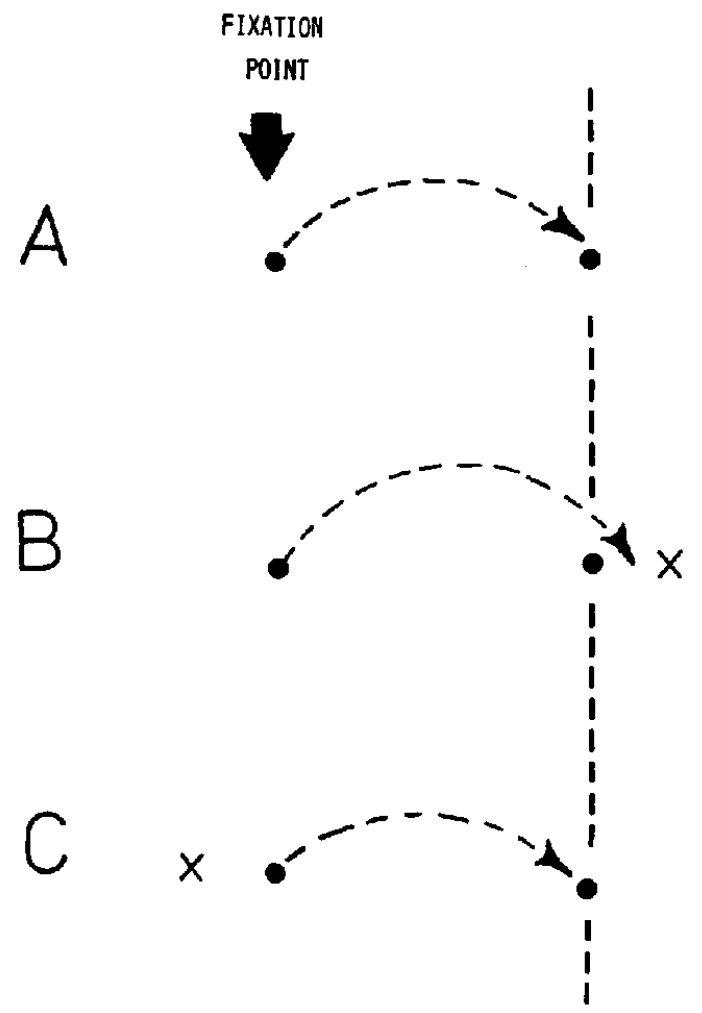

Figure 12. Effect of eye position in the array on eye movement readinesses. The eye movement held in readiness is longer than the unbiased movement, $A$, only in a situation such as $B$, where the saccade is pulled toward the extraneous stimulus $(X)$. In the situation where the extraneous stimulus is part of the configuration but not relevant for the eye movement held in readiness, $C$, no distortion in saccade length occurs.

the stimulus configuration. On the other hand, if the global configurational properties produce the perceptual distortion (which in turn later evokes the erroneous eye movements), the distortion in the perception of visual extent will exist, regardless of where in the stimulus array the eye dwells.

This notion was tested using the overestimation segment of the center of gravity illusion, similar to those shown in Figure 12. The distance between the two dots defining the horizontal extent was fixed at $8 \mathrm{~cm}$. For half of the trials, the observer's task was to maintain fixation on the leftmost dot in the configuration (indicated by arrows in Figure 12), and for the other half of the trials the observer was to maintain fixation on the rightmost dot in the array. An extraneous stimulus consisting of a red $X$ (with each arm equal to $5 \mathrm{~mm}$ ) could either be placed in proximity to the fixation point (as in Figure 12C) where it would be relatively irrelevant to the size of the eye movement held in readiness to fixate the other target dot delineating the test extent, or it could be placed in proximity to the target stimulus (as in Figure 12B) where its placement should bias the efferent readiness toward emission of an overly long saccadic eye movement. The distance between the extraneous stimulus and either the fixation point or the final target point was varied and could be either $2 \mathrm{~cm}$, $4 \mathrm{~cm}$, or $6 \mathrm{~cm}$ (plus a control condition with no extraneous stimulus). 


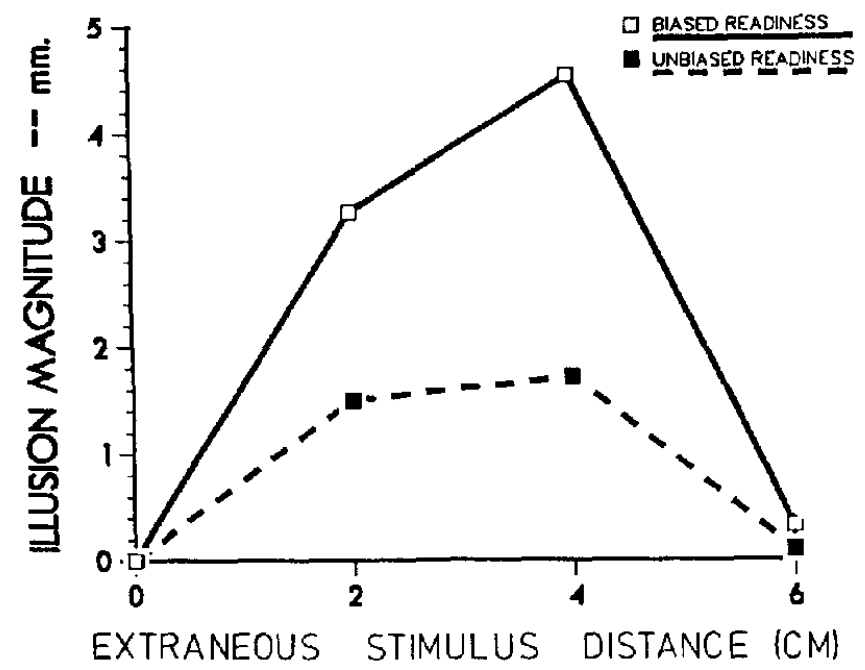

Figure 13. Data revealing a systematic change in the magnitude of the overestimation of linear extent as a function of the distance of the extraneous stimulus for conditions where the eye movement tendencies are biased (as in Figure 12B), but not for the same configuration where the eye is placed in a position where the eye movement readiness would not be biased (as in Figure 12C).

Subjects viewed the stimuli with their heads immobilized in a head and chin rest. In front of them was a small shelf, under which their hands rested, out of sight. Affixed to the underside of this shelf was a millimeter scale. Subjects made their perceptual judgments as to the length of the distance between the dots by holding their forefingers against this scale, separating them by a distance equivalent to the apparent test extent. Two measures were taken for each extraneous stimulus placement and fixation condition.

In the condition where the eye movements would be expected to be biased (Figure 12B), an overestimation of the target length, relative to that which should be obtained when no extraneous stimuli are present, would be expected, whereas in the situation where the placement of the extraneous stimulus should not bias the eye movement toward the distal target (Figure $12 \mathrm{C}$ ), no distortion, or at least an attenuated distortion, would be expected. Twelve observers were tested under these conditions, and the results are shown in Figure 13.

Notice that in the biased eye movement condition (such as Figure 12B shows), the magnitude of the illusion obtained is considerably greater than that obtained for the unbiased situation in which the placement of the target is next to the fixation (e.g., Figure 12C) as predicted by efferent considerations. The difference in illusion magnitude as a function of the location of the eye in the array is significant, $F(1,44)=7.51, p<.01$. Although the illusion is much greater in the biased placement, it is not completely absent in the irrelevant placement, probably because the eyes tend to drift off of the fixation point somewhat, hence evoking the necessity to saccade back to the initial fixation point and, hence, of course, the appropriate efferent readinesses toward overestimating the extent. Of particular interest is the fact that the size of the illusory distortion does vary quadratically with the distance between the target and the extrane- ous stimulus, as expected on the basis of the above reasoning. This quadratic component is significant for the condition in which the placement of the extraneous stimulus would be expected to bias the saccade toward an overly long eye movement, $F(1,44)=5.57, p<.05$, but does not achieve statistical signifcance in the condition where the placement of the extraneous stimulus is relatively irrelevant, $F(1,44)=2.16$. For the biased eye movement readiness conditions, the pattern of results is quite similar in magnitude and form to those depicted for the primary center of gravity illusion in Figure 5, while little effect is manifest for the unbiased eye movement condition.

The importance of these findings is that they seem to provide a release from the limitations associated with the correlational aspects of the data presented previously. These data clearly show that the existence of the predicted distortion does not depend solely on the configurational aspects of the array. Because eye position yaries the pattern of responses held in readiness, a theory in which the percept reflects the set of efferent readinesses should predict that the magnitude of an illusory distortion might also vary as a function of eye position in the array. This prediction suggests that the controlling sequence moves from the motoric to the perceptual in direction, rather than from the perceptual to the motoric. This seems to imbue the motoric components of the process with some priority, thus moving beyond the simple correlational loop that has characterized earlier work in this area.

\section{Demonstration 7: Attentional Factors}

An interesting issue that may be related to the preceding set of findings pertains to attentional considerations. When an individual is said to be paying attention to a given set of stimuli, one might hypothesize that what is really meant is that this individual is prepared, or ready to respond, to that set of stimuli. On the other hand, not paying attention to a given set of stimuli is equivalent to not having a set of efferent commands held in readiness for that particular stimulus. In other words, attending to or not attending to a given set of stimuli has some of the same components as perceiving or not perceiving a given set of stimuli, in that attention implies the evocation of a particular set of efferent readinesses. Operationally, this implies that an alternate way to illustrate the operation of efferent readinesses on perception would be to hold configurational properties constant and to shift only attention. If such attentional shifts produce changes in the perception of direction and extent, then they provide some convergent evidence for the present theoretical position.

A study conducted by Coren and Porac (1983) bears directly on this issue. It seems to show that by manipulating efferent readinesses, via the direction of an observer's attention, one can produce a systematic change in the amount of illusory distortion obtained in a variant of a classical illusion configuration. They used a configuration that contains both the over- and underestimated segments of the Mueller-Lyer illusion, presented in a confounded form. Using this array, they were able to demonstrate that the very existence and direction of the illusion depended on the attention paid to parts of the array (and hence, according to this analysis, the readiness to respond to parts of the array). 
A
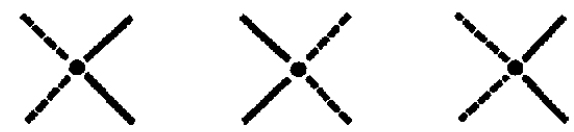

B
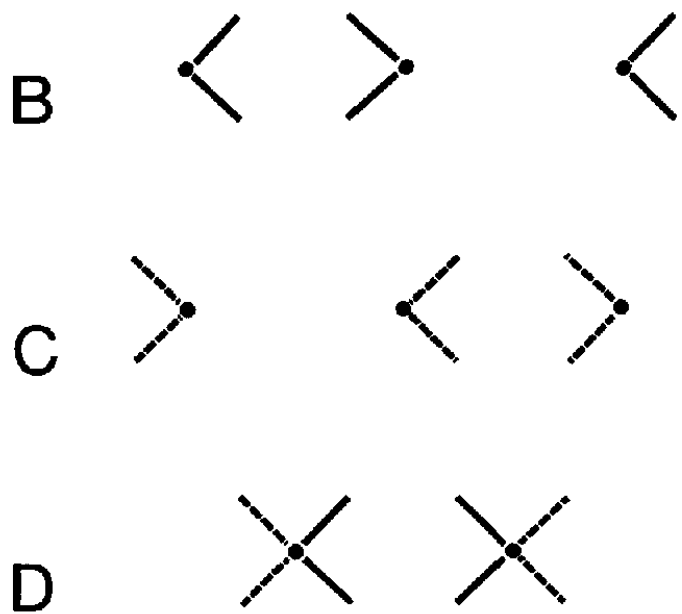

Figure 14. Composite figure (A) containing two variants of the MuellerLyer illusion (B and C). (Directing attention to the solid lines causes the distance between the dots on the left side to appear shorter than the distance between those on the right, whereas directing the attention to the broken lines reverses the illusion.) D: Simplified version, permitting only a single over- or underestimation as a function of which pair of wings is attended to (after Coren \& Porac, 1983).

To understand how attentional shifts relate to the formation of percepts embodying direction or extent, consider one configuration used by Coren and Porac (1983). This figure is shown as Figure 14A. According to any current theory of illusion formation, there is no reason to expect any distortion if subjects are asked to judge the distances between the dots centered in the three $X$-like components making up the figure, under normal viewing conditions. The distance between the right-hand pair and the left-hand pair of dots should be the same. To create an illusion in this array, one must first bias the set of efferent readinesses. This may be accomplished by an attentional shift. To see how this may be done, first notice that one half of each $X$ is composed of an angle made up of two solid lines, whereas the other half is composed of an angle made up of two broken lines. This device actually allows two opposite Mueller-Lyer illusions to be embedded in the same configuration. One consists of the Brentano variant of the illusion made up of solid lines (Figure 14B), where subjects would be expected to see the distance between the left pair of dots as shorter than the distance between the right pair of dots. The other is the inversion of this figure made up as broken lines (Figure 14C) where the reverse illusion occurs and the distance between the left pair of dots now appears longer.

It is easy to predict the Mueller-Lyer illusion seen in Figures $14 \mathrm{~B}$ and $14 \mathrm{C}$ on the basis of a recognition that the wings are merely serving as extraneous stimuli that shift the center of gravity of the stimuli region near the target stimulus (here the vertex) and bias the set of eye movements held in readiness, as analyzed earlier. In Figure 14A, however, because the two configurations coexist and the array of extraneous elements is symmetrical around the target stimulus, the biases tend to offset one another. However, by directing the observer's attention toward one or another of the sets of wings, the relative salience of one set of efferent readinesses relative to the others may be shifted. If the observer looks at Figure $14 \mathrm{~A}$ and simply confines his or her attention to the solid lines (thus ignoring the dashed lines), the same distortion found in Figure 14B immediately appears: The right horizontal extent appears longer than the left horizontal extent. Suppose the observer attends to the broken lines, ignoring the solid ones: The left horizontal extent appears to be longer than the right. Thus, shifting attention and, hence, shifting the set of responses held in readiness creates a systematic pattern of distortion in this formerly undistorted figure.

The existence of this attentionally dependent distortion was verified in formal measurements by Coren and Porac (1983), using a simplified version of this array shown in Figure 14D. This figure was either over- or underestimated according to whether the observer's attention was directed to the in-turned or the out-turned wings. This result is consistent with the idea that by direction of attention toward a particular set of nontarget stimuli, the set of efferent readinesses evoked to guide the eye movements over the array become biased in the direction of these stimuli. Because the final percept depends on the pattern of efference held in readiness, the percept is also biased, thus leading to the perceptual distortion.

Although the arguments above seem, at least at a logical level, to link efferent readinesses to shifts in attention, one would like some additional evidence verifying that the existence of the motoric bias is correlated with both the perceptual and the attentional biases. To do this, an array similar to that presented in Figure 14D was used. Although this latter figure employs broken and continuous lines, in the actual stimulus both halves of each $X$-like component were constructed of continuous lines, differing only in color (a green-yellow [GY] and a yellow-red [YR], with Munsell equivalents of 7.5 GY $7 / 10$ and 5 YR 7/10 respectively). Each of the wings of the $X$ were $2 \mathrm{~cm}$ long, and the target dot was black, $1 \mathrm{~mm}$ in diameter. The stimulus subtended a visual angle of $18^{\circ}$ and was viewed by eight subjects whose heads were immobilized in a head and chin rest. Horizontal eye movements were recorded using an infrared reflection system, which produces a resolution of approximately $30^{\prime}$ of arc under these conditions.

Subjects were required to systematically scan the two ends of the figure trying to fixate the black central target as accurately as possible. Attention was varied by directing subjects to pay attention to the wings of one color and to ignore the other. Twelve measurements were taken under each viewing instruction in mixed blocks of six.

Data were scored in terms of the length of the initial saccade, inasmuch as this is the best index of the eye movement held in readiness. The expectation is, of course, that the set of efferent readinesses will be affected by the attentional set of the observer. The eye movements should then be longer when attending to the outwardly turned wings (e.g., the broken lines in Figure 14D) than when attending to the inwardly turned wings (e.g., the solid lines in Figure 14D). This then would indicate a set 
of efferent readinesses that are consistent with the observer's judgment of apparent length under these conditions. This result was obtained. The mean length of the eye movement when attending to the outwardly turned wings was $19.6^{\circ}$. Contrast this to an average saccade length of only $16.7^{\circ}$ when attending to inturned wings. This difference is statistically significant, $t(7)=$ $3.14, p<.02$. The fact that the subjects were trying to fixate the target dot as accurately as possible, even given the attentional instructions, is demonstrated by the fact that in $94 \%$ of the trials, when the length of the saccade over- or undershot the target dot by $2^{\circ}$ or more, there was a corrective flick back toward the target within $350 \mathrm{~ms}$.

In this last demonstration data again are found that support an efferent component in the perception of extent. This demonstration indicates that by altering an observer's attentional set, the pattern of eye movements held in readiness can be systematically altered. As observation of Figure 14A and the data of Coren and Porac (1983) indicate, these same conditions also alter the perception of extent for this stimulus array. Thus, despite the fact that the visual stimulus has remained unchanged, changing the response readinesses changes the conscious percept. Taken in conjunction with the previous demonstration, this suggests that anything altering the motoric tendencies held in readiness when viewing a stimulus may also alter the perception of that stimulus.

\section{Efferent Contributions to Visual Perception}

This discussion was begun by pointing out that well over a century ago a number of psychologists began to entertain the notion that the incoming afferent stimulation and the outgoing motor innervation were part of a single continuous process with no point of separation between them. The evidence on which this was based involved a strong correlation between eye movements and the conscious perceptual representation of the stimulus, especially in illusion-type situations where the percept differed systematically from the physical reality along some dimension. These original notions have been modified here to suggest that the demands of the motoric system bias the information selected and its encoding. Afferent inputs are encoded into a set of efferent readinesses in order to guide subsequent motor activity. The conscious percept is based, at least in part, on this set of motoric responses held in readiness rather than on unprocessed, raw afferent sense data.

This research began with the observation that when observers are asked to view a target that appears in close proximity to extraneous stimuli, initial saccadic eye movements are somewhat biased toward the center of gravity of stimuli in the target vicinity. This eye movement bias results in predictable biases in perception. Those stimulus parameters that systematically bias the saccadic eye movement pattern were isolated, and a set of configurations that produced eye movements that manifested particular constant errors was created. Through extrapolating from the eye movement data, it was possible to predict a new set of illusions. Furthermore, the magnitude of these illusions varied parametrically with stimulus variations that affected the eye movement pattern. Five of the demonstration experiments that were presented not only showed the existence of a new class of illusions but also predicted some variants of classical visual- geometric illusions. In the last two demonstration experiments it was shown that the theory involves more than merely a correlation between eye movements and perception. Rather these demonstrations indicate that the manipulation of response readinesses seems to have a certain causal priority over the percept. In these, while keeping the configuration constant, it was shown that the magnitude of the predicted illusory distortions could be varied by altering the pattern of eye movement readiness through shifts in the point of fixation within the array or changes in the distribution of attention.

One point ought to be made as the analysis is concluded. It is not being suggested that the location of a stimulus, or the conscious representation of a distance, is solely determined by motoric factors, nor is the contention being made that the source of all of the distortion observed in visual-geometric illusions arises from a biased set of efferent readinesses held in consciousness. Such a contention would be far too strong and would be completely at variance with the work done over the past 15 years suggesting that a multiplicity of factors contribute to these nonveridical percepts, including refractive factors in the optical system of the eye, lateral neural interactions on the retina, cognitive contrast or assimilation, inappropriate processing of two-dimensional arrays as if they were three-dimensional, and a myriad of other processes occurring at various levels within the perceptual system. As has been pointed out in many places, the distortions arising from these various levels tend to additively combine to form the final illusory percept (Coren \& Girgus, 1978a, 1978b; Coren, Girgus, Ehrlichman, \& Hakstian, 1976; Coren \& Porac, 1983; Coren \& Ward, 1979). On the other hand, the findings presented here do indicate that there is some systematic motoric contribution to these distortions and, hence, also to the perception of direction and extent in more typical, nonillusion situations.

It is difficult, given the nature of most contemporary theorizing about perception, to accept the fact that nonvisual components, such as motoric responses held in readiness, may contribute to the metric properties of the final visual impression in consciousness. Muensterberg attempted to clarify this difficulty in 1914, when be wrote:

According to the popular view, a world of impressions and ideas
exist in us, entirely independent of our actions, and when they are
complete and perfect, they send their message to some motor appa-
ratus which carries out the order. Such a fancy must be entirely
reversed. In every moment, the motor situation decides the possi-
bilities in the sensory sphere. Our ideas are the product of a readi-
ness to act. . . We all perceive the world just as far as we are
prepared to react to it. . . We know that the stimulus coming
from different points of space awakes different reaction move-
ments. . . . May it not be that in all these cases, too, the character
of the processes in the motor spheres has influence on the preceding
sensorial excitement and gives a special shading to the mental im-
pression which leads to those actions? (pp. 140-142)

It is this "shading" of the percept, causing slight biases and misperceptions, that seems to be the nature of the motoric interaction. Awareness is a dynamic process. It can be viewed much like a busy railway station in which the incoming afferent stimulation is represented by the incoming trains and the efference is represented by the outgoing trains. Consciousness is the traffic pattern occurring within the station. Certainly the occurrences in such a large railway station can never be understood 
as long as only the incoming trains are studied and one does not pay attention to the outgoing trains. The directions taken by these incoming trains depend on the clearing and blocking of available tracks within the station and on the direction the outgoing trains are to take when they depart. Such activity will tend to bias the arrival platforms of any one train. In much the same way, what goes on in one's mental life depends on the clearing and blocking and switching of tracks for reaction. Both the input and the output affect the total response. Thus, although one must not conclude that knowledge of the motoric output is sufficient to determine what the final percept is, one must similarly recognize that knowledge of incoming stimuli will not suffice. Knowledge of both the afference and the resulting processing used to guide the efferent responses is required to obtain an accurate assessment of how one arrives at conscious impressions of the location of extents of objects in the visual world.

\section{References}

Auerbach, F. (1894). Erklaerung der Brentano's chen optischen Tauschung [Explanation of Brentano's optical illusion]. Zeitschrift Fur Psychologie, 7, 152-160.

Bain, A. (1855). The senses and the intellect. London: Longmans, Green.

Bierveliet, J. J., van. (1896). Nouvelles measures des illusions visuelles chez les adultes et chez les enfants [New measures of visual illusions in adults and children]. Revue Phiosophique, 41, 19-181.

Binet, A. (1895). La mesure des illusions visuelles chez les enfants [Measurements of illusions in children]. Revue Filosophique, 40, 11-25.

Brentano, F. (1892). Ueber ein optisches Paradoxen [A visual illusion]. Joumal of Psychology, 3, 249-258.

Bruell, J. H., \& Albee, G. W. (1955). Notes toward a motor theory of visual egocentral localization. Psychological Review, 62, 301-308.

Burnham, C. A. (1968). Decrement of the Mueller-Lyer illusion with saccadic and tracking eye movements. Perception and Psychophysics, 3, 424-426.

Cooper, L. A., \& Weintraub, D. J. (1970). Delboeuf-type in circle illusions: Interactions among luminance, temporal characteristics, and inducing figure variations. Journal of Experimental Psychology, 85, 75-82.

Coren, S. (1970). Lateral inhibition and geometrical illusions. Quarterly Journal of Experimental Psychology, 22, 274-278.

Coren, S. (1981). The interactions between eye movements and visual illusions. In D. F. Fisher, R. A. Monty, \& J. W. Senders (Eds.), Eye movements, cognition and visual perception (pp. 61-87). Hillsdale, NJ: Erlbaum.

Coren, S., Bradley, D. R., Hoenig, P., \& Girgus, J. S. (1975). The effect of smooth tracking and saccadic eye movements on the perception of size: The shrinking circle illusion. Vision Research, 15, 49-52.

Coren, S., \& Festinger, L. (1967). An alternative view of the "Gibson normalization effect." Perception and Psychophysics, 2, 621-626.

Coren, S., \& Girgus, J. S. (1978a). Seeing is deceiving: The psychology of visual illusions. Hillsdale, NJ: Erlbaum.

Coren, S., \& Girgus, J. S. (1978b). Visual illusions. In R. Held, H. Leibowitz, \& H-L. Teuber (Eds.), Handbook of sensory physiology: Perception (Vol. 8, pp. 549-568). Berlin: Springer-Verlag.

Coren, S., Girgus, J. S., Ehrlichman, H., \& Hakstian, A. R. (1976). An empirical taxonomy of visual illusions. Perception and Psychophysics, 20, 129-137.

Coren, S., \& Hoenig, P. (1972). Effect of non-target stimuli upon the length of voluntary saccades. Perceptual and Motor Skills, 34, 499508.

Coren, S., \& Porac, C. (1983). The creation and reversal of the Mueller-
Lyer illusion through attentional manipulation. Perception, 12, 4954.

Coren, S., \& Ward, L. M. (1979). Levels of processing in visual illusions: A combination and interaction of distortion-producing mechanisms. Joumal of Experimental Psychology: Human Perception and Performance, 5, 324-335.

Crovitz, H. F., \& Davies, W. (1962). Tendencies to eye movements and perceptual accuracy. Journal of Experimental Psychology, 63, 495498.

Delabarre, E. B. (1897). A method of recording eye movements. American Journal of Psychology, 9, 572-574.

DeSisto, M. J., \& Moses, F. L. (1968, September). Saccadic eye movement response to the Mueller-Lyer stimuli. Paper presented at the meeting of the Eastern Psychological Association, Washington, DC.

Dewar, R. E. (1967). Stimulus determinants of the magnitude of the Mueller-Lyer illusion. Perceptual and Motor Skills, 24, 708-710.

Ditchburn, R. W., \& Ginsborg, B. L. (1952). Vision with a stabilized retinal image. Nature, $170,36-37$.

Evans, C. R., \& Marsden, R. P. (1966). A study of the effect of perfect retinal stabilization on some well-known visual illusions, using the after-image as a method of compensating for eye movements. British Journal of Physiological Optics, 23, 242-248.

Festinger, L., Burnham, C. A., Ono, H., \& Bamber, D. (1967). Efference and the conscious experience of perception. Journal of Experimental Psychology Monographs, 74(4, Pt 2).

Festinger, L., White, C. W., \& Allyn, M. R. (1968). Eye movements and decrement in the Mueller-Lyer illusion. Perception and Psychophysics, 3, 376-382.

Findlay, J. M. (1980). The visual stimulus for saccadic eye movements in human observers. Perception, 9, 7-21.

Findlay, J. M. (1981). Local and global influences on saccadic eye movements. In D. F. Fisher, R. A. Monty, \& J. W. Senders (Eds.), Eye movements: Cognition and visual perception (pp. 171-181). Hillsdale, NJ: Erlbaum.

Gibson, J. J. (1979). The ecological approach to visual perception. Boston: Houghton Miffin.

Graham, N., Robson, J. G., \& Nachmias, J. (1978). Grating summation in fovea and periphery. Vision Research, 18, 815-825.

Hebb, D. O. (1949). The organization of behavior. New York: Wiley.

Heymans, G. (1896). Quantitative Untersuchungn ueber das optische Paradoxen [Quantitative investigations of a visual illusion]. Zeitschrift fur Psychologie, 9, 221-255.

Hicks, G. D., \& Rivers, W. H. R. (1908). The illusion of compared horizontal and vertical lines. British Journal of Psychology, 2, 243-260.

Hubel, D. H. (1978). Vision and the brain. Bulletin of the American Academy of Arts and Sciences, 31, 17-28.

Jacobson, E. (1938). Progressive relaxation. Chicago: University of Chicago Press.

Judd, C. H. (1905). The Mueller-Lyer illusion. Psychological Review Monographs, 7,(Suppl. 29), 55-82.

Kaufman, L., \& Richards, W. (1969). Spontaneous fixation tendencies for visual forms. Perception and Psychophysics, 5, 85-88.

Levy-Schoen, A. (1974). Le champ d'activité du regard: Donnes experimentales [Scope of active looking: Given experimentally]. L'année Psychologique, 74, 43-66.

Levy-Schoen, A., \& Rigaut-Renard, C. (1979). Pre-perception on activation motrice au cours du T.R. oculomoteur? [Preperceptual motor activation and the path of translational eye movements]. In J. Requin (Ed.), Anticipation et comportement. Paris: Editions du CNRS.

Lewis, E. O. (1909). Confluxion and contrast effects in the Mueller-Lyer illusion. British Journal of Psycholgy, 3, 21-41.

Lipps, T. (1897). Raumaustahetik und geometrisch-optische Taeuschungen [Framing effects on geometrical optical illusions]. Leipzig, East Germany: Barth. 
Lotze, R. (1852). Medicinisch psychologie [Medical psychology]. Leipzig, East Germany: Weidmann.

Max, L. W. (1935). An experimental study of the motor theory of consciousness. Journal of Comparative and Physiological Psychology, 19, 469-486.

Muensterberg, H. (1914). Psychology, General and Applied. New York: Appleton.

Nakagawa, D. (1958). Mueller-Lyer illusion and retinal induction. Psychologia, 1, 167-174.

Oppel, J. J. (1854-1855). Ueber geometrisch-optisch tauschungen [Geometrical optical illusions]. Jarhresbericht des Frankfurter Vereins, 37-47.

Piaget, J., \& Bang, V. (1961). Comparison des mouvents ocularies et des centrations du regard chez l'enfant, et chez l'adulte [Comparison of eye movements and viewing centrations in children and adults]. $A r$ chives de Psychologie Geneve, 38(Whole No. 150), 167-200.

Pollack, R. H. (1970). Mueller-Lyer illusion: Effect of age, lightness, contrast and hue. Science, 170, 93-95.

Pritchard, R. M. (1958). Visual illusions viewed as stabilized retinal images. Quarterly Journal of Experimental Psychology, 10, 77-81.

Restle, F., \& Decker, J. (1977). Size of the Mueller-Lyer illusion as a function of its dimensions: Theory and data. Perception and Psychophysics, 21, 489-503.

Richards, W., \& Kaufman, L. (1969). "Center-of-gravity" tendencies for fixations and flow patterns. Perception and Psychophysics, 5, 8183.

Robinson, J. O. (1972). The psychology of visual illusion. London: Hutchinson.

Skinner, B. F. (1974). About behaviorism. New York: Knopf.
Smith, S. M., Brown, H. D., Toman, J. E. P., \& Goodman, L. S. (1947). The lack of cerebral effects of d-tubocurarine. Anesthesiology, 8,1 14.

Sperry, R. (1952). Neurology and the mind-brain problem. American Scientist, 40, 291-312.

Stratton, G. M. (1906). Symmetry, linear illusions, and the movements of the eye. Psychological Review, 13, 81-96.

Taylor, J. G. (1962). The behavioral basis of perception. New Haven, CT: Yale University Press.

Virsu, V. (1971). Tendencies to eye movement and misperception of curvature, direction and length. Perception and Psychophysics, 9, 6572.

Washburn, M. F. (1916). Movement and mental imagery. Boston: Houghton Mifflin.

Watson, J. B. (1930). Behaviorism. Chicago: University of Chicago Press.

Weisstein, N., \& Harris, C. S. (1980). Masking and the unmasking of distributed representations in the visual system. In C. S. Harris (Ed.), Visual coding and adaptability (pp. 317-364). Hillsdale, NJ: Erlbaum.

White, K. G. (1972). Implicit contours in the Zoellner illusion. American Journal of Psychology, 85, 421-424.

Wundt, W. (1897). Outlines of psychology (C. H. Judd, Trans.). Leipzig, East Germany: Engelmann.

Yarbus, A. L. (1967). Eye movements and vision. New York: Plenum Press.

Received May 31, 1985

Revision received November 20, 1985

\section{Instructions to Authors}

Authors should prepare manuscripts according to the Publication Manual of the American Psychological Association (3rd ed.). All manuscripts must include an abstract of 75-100 words typed on a separate sheet of paper. Typing instructions (all copy must be double-spaced) and instructions on preparing tables, figures, references, metrics, and abstracts appear in the Manual. Also, all manuscripts are subject to editing for sexist language.

APA policy prohibits an author from submitting the same manuscript for concurrent consideration by two or more journals. APA policy also prohibits duplicate publication, that is, publication of a manuscript that has already been published in whole or in substantial part in another journal. Authors of manuscripts submitted to APA journals are expected to have available their raw data throughout the editorial review process and for at least 5 years after the date of publication.

Authors will be required to state in their initial submission letter or sign a statement that they have complied with APA ethical standards in the treatment of their sample, human or animal. (A copy of the APA Ethical Principles may be obtained from the APA Ethics Office, 1200 17th Street, N.W., Washington, DC 20036.)

Anonymous reviews are optional, and authors who wish anonymous reviews must specifically request them when submitting their manuscripts. Each copy of a manuscript to be anonymously reviewed should include a separate title page with authors' names and affiliations, and these should not appear anywhere else on the manuscript. Footnotes that identify the authors should be typed on a separate page. Authors should make every effort to see that the manuscript itself contains no clues to their identities.

Manuscripts should be submitted in quadruplicate. All copies should be clear, readable, and on paper of good quality. A dot matrix or unusual typeface is acceptable only if it is clear and legible. Dittoed and mimeographed copies will not be considered. Authors should keep a copy of the manuscript to guard against loss. Mail manuscripts to the Editor, Martin L. Hoffman, Psychological Review, Department of Psychology, New York University, 6 Washington Place, 4th floor, New York, New York 10003, according to the instructions provided above. 